\title{
4-Component Relativistic Calculations in
}

Solution with the Polarizable Continuum Model

of Solvation: Theory, Implementation and

\section{Application to the Group 16 Dihydrides $\mathrm{H}_{2} \times(X$$$
=0, \mathrm{~S}, \mathrm{Se}, \mathrm{Te}, \mathrm{Po})
$$

Roberto Di Remigio, ${ }^{*}+, \|$ Radovan Bast, ${ }^{\dagger,}, \boldsymbol{\Phi}, \perp$ Luca Frediani, ${ }^{\dagger}$ and Trond Saue ${ }^{*, \S}$

Department of Chemistry, Centre for Theoretical and Computational Chemistry, University of Tromsø, N-9037 Tromsø, Norway, Department of Theoretical Chemistry and Biology,

School of Biotechnology, Royal Institute of Technology, AlbaNova University Center,

S-10691 Stockholm, Sweden, PDC Center for High Performance Computing, Royal

Institute of Technology, S-10044 Stockholm, Sweden, and Laboratoire de Chimie et

Physique Quantiques (UMR 5626) CNRS/Université de Toulouse 3 (Paul Sabatier) 118

route de Narbonne, 31062 Toulouse, France

E-mail: roberto.d.remigio@uit.no; trond.saue@irsamc.ups-tlse.fr 


\begin{abstract}
We present a formulation of 4-component relativistic self-consistent field (SCF) theory for a molecular solute described within the framework of the polarizable continuum model (PCM) for solvation. The linear response function for a 4-component PCM-SCF state is also derived, as well as the explicit form of the additional contributions to the first-order response equations. The implementation of such a 4-component PCMSCF model, as carried out in a development version of the DIRAC program package, is documented. In particular, we present the newly developed application programming interface (API) PCMSolver used in the actual implementation with DIRAC. To demonstrate the applicability of the approach we present and analyze calculations of solvation effects on the geometries, electric dipole moments and static electric dipole polarizabilities for the group 16 dihydrides $\mathrm{H}_{2} \mathrm{X}(\mathrm{X}=\mathrm{O}, \mathrm{S}, \mathrm{Se}, \mathrm{Te}, \mathrm{Po})$.
\end{abstract}

\title{
Keywords
}

Continuum solvation, relativistic quantum chemistry, electrostatic potential, modular programming, linear response, polarizability, dipole moment

\footnotetext{
${ }^{*}$ To whom correspondence should be addressed

${ }^{\dagger}$ Department of Chemistry, Centre for Theoretical and Computational Chemistry, University of Troms $\varnothing$, N-9037 Troms $\varnothing$, Norway

${ }_{\ddagger}^{\ddagger}$ Department of Theoretical Chemistry and Biology, School of Biotechnology, Royal Institute of Technology, AlbaNova University Center, S-10691 Stockholm, Sweden

ฯPDC Center for High Performance Computing, Royal Institute of Technology, S-10044 Stockholm, Sweden

${ }^{\S}$ Laboratoire de Chimie et Physique Quantiques (UMR 5626) CNRS/Université de Toulouse 3 (Paul Sabatier) 118 route de Narbonne, 31062 Toulouse, France

"Previous address: Dipartimento di Chimica e Chimica Industriale, Università di Pisa, via Risorgimento 35, 56126 Pisa, Italy

${ }^{\perp}$ Previous address: Laboratoire de Chimie et Physique Quantiques (UMR 5626) CNRS/Université de Toulouse 3 (Paul Sabatier) 118 route de Narbonne, 31062 Toulouse, France
} 


\section{Introduction}

Paul Adrien Maurice Dirac, who developed the relativistic wave equation for the electron, stated that relativistic effects would be of "no importance in the consideration of atomic and molecular structure and ordinary chemical reactions" "1]. Trusting Dirac's words, most of the successful development in quantum chemistry has been based on nonrelativistic quantum mechanics, but Dirac's statement has been proven incorrect ${ }^{2}$. From a chemist's point of view, this is not obvious. Relativistic corrections depend on the ratio between particle speed and the speed of light $\underline{3}$. Since valence electrons have small kinetic energies, one would think, as did Dirac, that chemical bonding and structure would be unaffected. After all, most chemical phenomena take place at energies well below the relativistic regime: the rest energy of an electron $\left(m_{\mathrm{e}} c^{2}\right)$ is half a mega-electron volt, while most chemical processes occur on an energy scale of few electronvolts.

This is not entirely true: it soon became clear in the history of theoretical chemistry, that a nonrelativistic theory could not explain certain trends in observed properties. As recently reviewed by Pyykkö, relativistic effects play a prominent role, especially in inorganic chemistry ${ }^{4}$. Periodic trends, such as the increase in atomic dimensions and ionization potentials, may be broken for the heaviest elements of the Periodic Table. Many examples are known, the most well-known maybe being the relativistic origin of the color of gold 5 .

In a less known part of his famous quote, ${ }^{1}$ Dirac advocates the development of numerical methods to solve the Schrödinger's equation. Although this effort has largely been undertaken for isolated molecules, the presence of solvent still poses a formidable challenge. On the other hand, the ability to account, at least qualitatively, for environment effects is fundamental in all experimental branches of chemistry: structures, energies and reaction barriers, as well as spectroscopic observables, are greatly influenced by environment effects. Despite the large number of reactions known to happen in the solid state and in gas phase, we can safely state that most chemistry happens in solution ${ }^{6}$. The theoretical treatment of environment effects suffers from a dimensionality problem: even the most simplified picture of 
the system under consideration would require taking into account 500-1000 atoms, at least. Direct application of quantum chemistry methods is therefore impossible and not even desirable for such systems. In problems with a high dimensionality, the microscopic detail in the physical description cannot account for the macroscopic behavior of the system $\underline{7}\left[\frac{9}{}\right.$. Models must be devised to overcome the dimensionality "disease".

It is customary to divide the models proposed into two broad classes, according to the microscopic description of the solvent these models give. Continuum (or implicit) models, deal explicitly only with the degrees of freedom associated with the solute, while replacing the solvent with a structureless continuum characterized by its bulk properties ${ }^{10111}$. Discrete (or explicit) models, treat degrees of freedom associated with the solvent molecules and the solute explicitly. The two sets of coordinates can be treated at different levels of theory. The quantum mechanics/molecular mechanics (QM/MM) family of methods is a notable example 12 .14. QM/MM and continuum methods can also be combined $[15,17$, to achieve a faithful, yet cost-effective strategy to reproduce solvent effects.

It is very well recognized that both solvent and relativistic effects can play an important role in the chemistry of heavy-element containing species. The field of actinide chemistry is in this respect a notable example ${ }^{18}$. Computational actinide chemistry is a very active field $\frac{19 \mid 20}{}$ and proper treatment of relativistic and solvent effects is mandatory: gaining insight about actinide species from experiment can be rather difficult or not practicable due to the safety and security hazards their radioactivity poses.

Relativistic and solvent effects also play an important role in the accurate prediction of NMR parameters of heavy-element containing species. Chemical shifts and indirect spinspin coupling constants show great sensitivity to the chemical environment of the probe nucleus 21 . Such observables are excellent benchmarks for assessing the accuracy of computational models for the inclusion of relativistic and solvent effects.

Despite numerous studies in the literature 2 both relativistic and solvent effects in a manner that is both efficient and accurate is not 
yet available. Many applications in the calculation of structures and energetics of heavyelement containing species make use of effective core potentials (ECPs) in a DFT or wave function theory framework $k^{25}$. In other studies, the more refined Zeroth-Order Regular Approximation (ZORA) Hamiltonian ${ }^{33}$ has been used, either in the scalar, spin-free form or in a 2-component framework, including spin-orbit effects 34 39. The second-order DouglasKroll-Hess (DKH2) in spin-free form, has also been applied 40 . In the above cited studies continuum approaches, notably the conductor-like screening model (COSMO) 4143 , have been adopted for the inclusion of solvent effects, in many cases complementing a cluster approach, treating the first solvation shell explicitly at the same level of theory as the solute. QM/MM studies on uranium complexes in water solution $\underline{44 \mid 45}$ and copper in plastocyanins ${ }^{46}$ have also appeared. Chaumont and Wipff ${ }^{47}$ presented a study of uranyl and europium solvation in room-temperature ionic liquids based on MD simulations combined with QM calculations on selected snapshots.

The calculation of NMR parameters for heavy-element containing compounds covers a large part of the existing literature. Autschbach and co-workers have presented extensive studies of shieldings and couplings for $\mathrm{Pt}-\mathrm{Tl}, \mathrm{Pt}$ and $\mathrm{Hg}$ containing species, alternatively

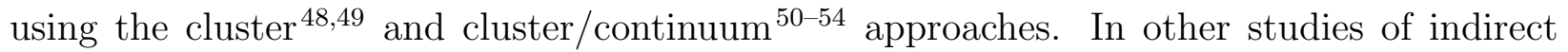
spin-spin coupling constants by the same group ${ }^{55556}$, Born-Oppenheimer molecular dynamics (BOMD) simulations were used to account for specific solvent effects.

In all aforementioned studies, bulk solvent effects, described by use of a continuum model such as COSMO, were found to be extremely important in order to achieve a qualitatively accurate description of the phenomena of interest. Remarkably, such a conclusion was reached for aprotic $\frac{23}{23}$ and protic ${ }^{\sqrt[50 \mid 54]{54}}$ solvents alike. However, a purely continuum description might in some cases not be adequate, to capture all relevant solute-solvent effects, requir-

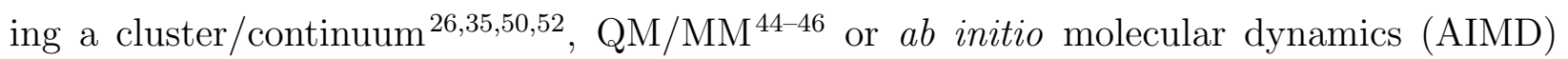
approach $\underline{54}[58$.

All the studies currently available in the literature emphasize the importance of both rela- 
tivistic and solvent effects. However, with the exception of a recent frozen density embedding subsystem approach? ?, a scheme whereby the 4-component Dirac-Coulomb Hamiltonian is coupled to an environment is not yet available. In the present contribution we report the interfacing of the polarizable continuum model $(\mathrm{PCM})^{59}$, a continuum solvation model, with a 4-component relativistic description of the solute. The equations derived are based on the self-consistent field (SCF) approximation for the wave function, either Hartree-Fock (HF) or Kohn-Sham density-functional theory (KS-DFT). The linear response (LR) function for the calculation of static second-order properties is also derived. We furthermore present a working implementation, making use of a modular programming paradigm (vide infra).

Due to the theoretical similarity between the PCM, more refined polarizable molecular mechanics $\frac{13144}{13}$ and even three-layer methodologies ${ }^{15}-17$, this work constitutes a starting point for more accurate models for the inclusiont of environment effects in computational procedures based on relativistic Hamiltonians.

The remainder of the paper is organized as follows. In Section 2 , a detailed presentation of the theory underlying the 4-component implementation of the PCM-SCF and LR-PCMSCF algorithms is presented. In Section 3, our modular approach to the implementation in the DIRAC program ${ }^{60}$ is discussed, with particular emphasis on the recently developed PCMSolver application programming interface (API) ${ }^{61}$. As a first application, we present in Section 4 geometries and electric properties of the group 16 dihydrides, with and without solvent effects included. Conclusions and perspectives for this work are presented in Section 5. SI-based atomic units ${ }^{62}$ will be employed throughout the paper $\left(\hbar=m_{e}=e=\frac{1}{4 \pi \varepsilon_{0}}=1\right)$, electron mass and charge will however be always specified explicitly in the equations. We will denote the identity matrix in a $N$-dimensional space by $I_{N}$. 


\section{Theory}

Sections 2.1 and 2.2 briefly summarize 4-component Hamiltonians and the IEF-PCM formalism, respectively. These are our points of departure for the formulation of the coupling of a 4-component description of the solute with a continuum solvation model, presented in Sections 2.3 and 2.4 .

\subsection{4-component Hamiltonians}

As recently emphasized by Saue ${ }^{63}$, the molecular electronic Hamiltonian may be written in the general form:

$$
H_{0}=\sum_{i}^{N} h\left(\boldsymbol{r}_{i}\right)+\frac{1}{2} \sum_{i, j=1}^{N} g\left(\boldsymbol{r}_{i}, \boldsymbol{r}_{j}\right)+V_{\mathrm{NN}} .
$$

i. e. as the sum of one- and two-electron parts together with a scalar shift $V_{\mathrm{NN}}$ due to the classical repulsion of clamped nuclei in the Born-Oppenheimer approximation. This general form remains valid whether relativity is being considered or not. Much alike the concept of nonrelativistic model chemistries one can consider relativity as the third dimension of quantum chemistry. The actual Hamiltonian model and the corresponding "rung" in the hierarchy of relativistic model chemistries is completely specified once the one- and twoelectron operators have been fixed.

In 4-component relativistic quantum chemistry, the one-electron Hamiltonian is taken to be the Dirac one-electron Hamiltonian ${ }^{364}$. In the field of the clamped nuclei and in the absence of any other external field, we have:

$$
h_{\mathrm{D}}=\left(\begin{array}{cc}
V_{\mathrm{Ne}} I_{2} & c(\boldsymbol{\sigma} \cdot \boldsymbol{p}) \\
c(\boldsymbol{\sigma} \cdot \boldsymbol{p}) & \left(V_{\mathrm{Ne}}-2 m_{e} c^{2}\right) I_{2}
\end{array}\right) ; \quad V_{\mathrm{Ne}}=-e \sum_{A} \int \mathrm{d} \boldsymbol{r}^{\prime} \frac{\rho_{A}\left(\boldsymbol{r}^{\prime}\right)}{\left|\boldsymbol{r}-\boldsymbol{r}^{\prime}\right|}
$$

where $c$ is the speed of light. The nuclear charge distributions may be chosen to have a finite spatial extent, as apparent from the above definition of the clamped-nuclei attractive potential. Furthermore notice that the form of $V_{\mathrm{Ne}}$ is the same as in nonrelativistic theory, 
but its physical content in the relativistic context is markedly different, in that it also contains the spin-orbit interaction $\underline{63}$. Finally, $\boldsymbol{\sigma}$ is a vector containing the Pauli spin matrices ${ }^{3}$.

In the relativistic context, the formulation of the two-electron interaction requires careful consideration. The full history of the interacting particles is required for a complete description of the two-electron interaction, and no closed expression is available for use in the electronic Hamiltonian. Rather, a perturbation expansion of the full two-electron interaction in orders of $c^{-2}$ can be used ${ }^{3}$

$$
\begin{aligned}
g\left(\boldsymbol{r}_{1}, \boldsymbol{r}_{2}\right) & \simeq g_{\mathrm{C}}\left(\boldsymbol{r}_{1}, \boldsymbol{r}_{2}\right)+g_{\mathrm{G}}\left(\boldsymbol{r}_{1}, \boldsymbol{r}_{2}\right)+g_{\text {gauge }}\left(\boldsymbol{r}_{1}, \boldsymbol{r}_{2}\right) \\
& =\frac{I_{4} \cdot I_{4}}{r_{12}}-\frac{c \boldsymbol{\alpha}_{1} \cdot c \boldsymbol{\alpha}_{2}}{c^{2} r_{12}}-\frac{\left(c \boldsymbol{\alpha}_{1} \cdot \boldsymbol{r}_{12}\right)\left(c \boldsymbol{\alpha}_{2} \cdot \boldsymbol{r}_{12}\right)}{c^{2} r_{12}^{3}}
\end{aligned}
$$

While the Coulomb term $g_{\mathrm{C}}$ represents the well-known charge-charge interaction, the Gaunt term $g_{\mathrm{G}}$ introduces a current-current interaction. A Foldy-Wouthuysen transformation $\underline{65}$ achieves reduction to 2-component form unveiling the physical content of these terms $\frac{66 \mid 67}{6}$. The Coulomb and Gaunt terms give rise to spin-orbit interaction of spin-same-orbit and spin-other-orbit type, respectively. The Gaunt term also carries the full spin-spin interaction, whereas the gauge-dependent term $g_{\text {gauge }}$ of Eq. (3) must be included for the full orbit-orbit interaction.

Different Hamiltonians to be used in relativistic molecular electronic-structure theory are built from the one-electron part in Eq. (2) and the two-electron interaction in Eq. (3), truncated at a suitable order in $c^{-2}$. Keeping only the Coulomb term gives rise to the Dirac-Coulomb (DC) Hamiltonian for an $N$-electron system:

$$
H_{\mathrm{DC}}=\sum_{i=1}^{N} h_{\mathrm{D}}\left(\boldsymbol{r}_{i}\right)+\frac{1}{2} \sum_{i, j=1}^{N} g_{\mathrm{C}}\left(\boldsymbol{r}_{i}, \boldsymbol{r}_{j}\right)
$$

It must be pointed out that the one-electron Hamiltonian in Eq. (2) is unbounded from below, so that one might expect that building a variational theory on it would not be possible $e^{68 / 69}$. The spectrum of the free-particle Dirac Hamiltonian ${ }^{3 / 64}$ features two branches, one 
above $m c^{2}$ and one below $-m c^{2}$, and this is a consequence of the fact the Dirac equation describes both electrons and positrons, as might be seen by a charge-conjugation transformation. In quantum electrodynamics (QED), the negative branch of the spectrum is reinterpreted so as to describe positrons with positive energy and charge opposite to that of the electrons. In relativistic quantum chemistry such a reinterpretation is not made, rather the orbitals of negative energy are treated as an orthogonal complement to the observable positive-energy solutions. A more detailed discussion of this point is postponed until the derivation of the 4-component PCM-SCF equations in Section 2.3.

Dyall showed that it is possible to reformulate the Dirac one-electron Hamiltonian in the molecular field ${ }^{70}$ as a sum of spin-dependent and spin-independent terms. Exploiting Dirac's identity and the coupling between large and small components of a 4-component spinor one can write:

$$
(\boldsymbol{\sigma} \cdot \boldsymbol{p}) V_{\mathrm{Ne}}(\boldsymbol{\sigma} \cdot \boldsymbol{p})=\boldsymbol{p} V_{\mathrm{Ne}} \cdot \boldsymbol{p}+\mathrm{i} \boldsymbol{\sigma} \cdot\left(\boldsymbol{p} V_{\mathrm{Ne}} \times \boldsymbol{p}\right) .
$$

By dropping the second, spin-dependent, term one obtains the spin-free form of the Dirac equation. Finally, it is possible, with analogous manipulations, to obtain a 4-component nonrelativistic wave equation: the Lévy-Leblond equation ${ }^{71}$. Using Dirac's relation $(\boldsymbol{\sigma} \cdot \boldsymbol{p})^{2}=$ $p^{2}$, the Lévy-Leblond equation is found to be equivalent to the Schrödinger equation.

In the rest of the paper, whenever referring to an Hamiltonian $H_{0}$ we might refer either to the Dirac-Coulomb, the Spinfree or the Lévy-Leblond Hamiltonian.

\section{$2.2 \quad$ IEF-PCM}

The polarizable continuum model is one of the most general continuum models available nowadays. In its Integral Equation Formulation (IEF-PCM) ${ }^{72}$ it can be used to model environments of different nature and complexity, such as isotropic solutions, liquid crystals and ionic liquids. In the case of isotropic solutions, the solvent is represented by a homogeneous, dielectric medium with relative permittivity $\varepsilon_{r}$ that is polarized by the molecular solute 
placed in a cavity $C$, with boundary $\partial C$, built in the bulk of the dielectric and modeled on the solute's geometry. The Poisson's Equation, with suitable boundary conditions, relates the electrostatic potential $\psi$ in the whole space to the solute's charge density $\rho$, supposed to be contained entirely inside the cavity:

$$
\begin{aligned}
-\nabla^{2} \psi(\boldsymbol{r}) & =4 \pi \rho(\boldsymbol{r}) \quad \forall \boldsymbol{r} \in C \\
-\varepsilon_{r} \nabla^{2} \psi(\boldsymbol{r}) & =0 \quad \forall \boldsymbol{r} \notin C \\
\psi_{\mathrm{i}}(\boldsymbol{s})-\psi_{\mathrm{e}}(\boldsymbol{s}) & =0 \quad \forall \boldsymbol{s} \in \partial C \\
\left.\frac{\partial \psi}{\partial \boldsymbol{n}}\right|_{\mathrm{i}}-\left.\varepsilon_{r} \frac{\partial \psi}{\partial \boldsymbol{n}}\right|_{\mathrm{e}}=0 & \forall \boldsymbol{s} \in \partial C
\end{aligned}
$$

where the subscripts $\mathrm{i}$ and e denote regions inside and outside the cavity, respectively, and $\boldsymbol{n}$ is the outward pointing normal vector.

To solve Poisson's problem, we rewrite the electrostatic potential as the sum of the molecular electrostatic potential and a reaction potential:

$$
\psi=\phi+\xi=\int_{C} \mathrm{~d} \boldsymbol{r}^{\prime} \frac{\rho\left(\boldsymbol{r}^{\prime}\right)}{\left|\boldsymbol{r}-\boldsymbol{r}^{\prime}\right|}+\int_{\partial C} \mathrm{~d} \boldsymbol{s} \frac{\sigma(\boldsymbol{s})}{|\boldsymbol{r}-\boldsymbol{s}|}
$$

where the charge density $\rho$ is the sum of nuclear and electronic contributions

$$
\rho(\boldsymbol{r})=\rho_{\mathrm{N}}(\boldsymbol{r})+\rho_{\mathrm{e}}(\boldsymbol{r}) .
$$

The reaction potential is expressed in terms of an Apparent Surface Charge (ASC) distribution $\sigma$ over the cavity boundary. To find the ASC we exploit the formalism of integral equations ${ }^{72173}$ which allows us to recast a problem in the whole Euclidean space $\mathbb{R}^{3}$ to a problem on a closed subset of $\mathbb{R}^{2}$, namely:

$$
\sigma(\boldsymbol{s})=\int_{\partial C} \mathrm{~d} \boldsymbol{s}^{\prime} \kappa\left(\boldsymbol{s}, \boldsymbol{s}^{\prime}\right) \phi\left(\boldsymbol{s}^{\prime}\right)=-\mathcal{T}^{-1}\left(\varepsilon_{r}\right) \mathcal{R} \phi(\boldsymbol{s})
$$


where the integral operators are defined in terms of the components of the Calderón projector

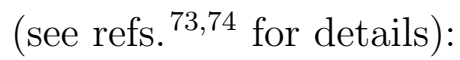

$$
\begin{aligned}
\mathcal{T} & =\left[2 \pi\left(\frac{\varepsilon_{r}+1}{\varepsilon_{r}-1}\right)-\mathcal{D}\right] \mathcal{S} \\
\mathcal{R} & =2 \pi-\mathcal{D} \\
\mathcal{S} f(\boldsymbol{s}) & =\int_{\partial C} f(\boldsymbol{s}) \frac{1}{\left|\boldsymbol{s}-\boldsymbol{s}^{\prime}\right|} \mathrm{d} \boldsymbol{s}^{\prime} \\
\mathcal{D} f(\boldsymbol{s}) & =\int_{\partial C} f(\boldsymbol{s}) \frac{\left(\boldsymbol{s}-\boldsymbol{s}^{\prime}\right) \cdot \boldsymbol{n}\left(\boldsymbol{s}^{\prime}\right)}{\left|\boldsymbol{s}-\boldsymbol{s}^{\prime}\right|} \mathrm{d} \boldsymbol{s}^{\prime}
\end{aligned}
$$

It can be proven that the $-\mathcal{T}^{-1}\left(\varepsilon_{r}\right) \mathcal{R}$ integral operator is self-adjoint due to the properties of the Calderón's projector components involved in its definition ${ }^{72 / 73}$.

The polarization energy, i.e. the energy contribution due to the interaction of the molecular electrostatic potential and the induced ASC, is given as:

$$
U_{\mathrm{pol}}[\rho]=\int_{\mathbb{R}^{3}} \mathrm{~d} \boldsymbol{r} \int_{\partial C} \mathrm{~d} \boldsymbol{s} \frac{\rho(\boldsymbol{r}) \sigma(\boldsymbol{s})}{|\boldsymbol{r}-\boldsymbol{s}|}=\int_{\mathbb{R}^{3}} \mathrm{~d} \boldsymbol{r} \int_{\mathbb{R}^{3}} \mathrm{~d} \boldsymbol{r}^{\prime} \rho(\boldsymbol{r}) M\left(\boldsymbol{r}, \boldsymbol{r}^{\prime}\right) \rho\left(\boldsymbol{r}^{\prime}\right)
$$

where we have

$$
M\left(\boldsymbol{r}, \boldsymbol{r}^{\prime}\right)=\int_{\partial C} \mathrm{~d} \boldsymbol{s} \int_{\partial C} \mathrm{~d} \boldsymbol{s}^{\prime} \frac{\kappa\left(\boldsymbol{s}, \boldsymbol{s}^{\prime}\right)}{|\boldsymbol{r}-\boldsymbol{s}|\left|\boldsymbol{r}^{\prime}-\boldsymbol{s}^{\prime}\right|} .
$$

The actual solution of Eq. (9) is achieved by means of a Boundary Element Method (BEM), i. e. by discretization of the cavity boundary with finite elements ${ }^{75}$. The surface is partitioned into $N_{\text {ts }}$ curvilinear triangles, called tesserae, with area $a_{I}$ and representative point $\boldsymbol{s}_{I}$. We assume that both the potential and ASC are constant on each tessera, so that their discrete representation are vectors of dimension $N_{\mathrm{ts}}$ with elements:

$$
v_{I}=\phi\left(\boldsymbol{s}_{I}\right) a_{I} \quad q_{I}=\sigma\left(\boldsymbol{s}_{I}\right) a_{I} .
$$


The $\boldsymbol{q}$ and $\boldsymbol{v}$ vectors are now related by a matrix equation:

$$
\boldsymbol{q}=\boldsymbol{K} \boldsymbol{v}
$$

where the response matrix $\boldsymbol{K}$ is the representation in the chosen discrete basis of the operator $-\mathcal{T}^{-1}\left(\varepsilon_{r}\right) \mathcal{R}$. The polarization energy is accordingly expressed as

$$
U_{\mathrm{pol}}=\boldsymbol{v} \cdot \boldsymbol{q}=\boldsymbol{v}^{\dagger} \boldsymbol{K} \boldsymbol{v}
$$

It is sometimes useful to partition the molecular solute electrostatic potential into a nuclear and an electronic component:

$$
\boldsymbol{v}=\boldsymbol{v}^{\mathrm{N}}+\boldsymbol{v}^{\mathrm{e}}
$$

so that a similar partition applies for the ASC:

$$
\boldsymbol{q}=\boldsymbol{q}^{\mathrm{N}}+\boldsymbol{q}^{\mathrm{e}}
$$

The polarization energy can then be expanded as

$$
U_{\mathrm{pol}}=U_{\mathrm{NN}}+U_{\mathrm{Ne}}+U_{\mathrm{eN}}+U_{\mathrm{ee}}=U_{\mathrm{NN}}+2 U_{\mathrm{eN}}+U_{\mathrm{ee}}
$$

where $U_{x y}(x, y=\mathrm{e}, \mathrm{N})$ is the interaction between the $x$ charge distribution and the $y$ induced apparent surface charge and we have used the fact that $U_{\mathrm{Ne}}=U_{\text {eN }}$, since $\mathcal{T}^{-1} \mathcal{R}$ is self-adjoint. 


\subsection{4-component PCM-SCF Theory}

In the presence of a solvent modeled as a classical continuum the Hamiltonian given in Eq. (1) must be corrected by a suitable PCM operator

$$
H_{\mathrm{eff}}=H_{0}+V_{\sigma \rho}[\rho]
$$

in which the PCM operator $V_{\sigma \rho}$ (vide infra) depends, linearly, on the solute density. The perturbation is thus state-specific and introduces a nonlinearity into the quantum mechanical problem. The functional to be minimized is accordingly $\underline{76}$ :

$$
G[\Psi]=\frac{\left\langle\Psi\left|H_{0}+\frac{1}{2} V_{\sigma \rho}[\rho]\right| \Psi\right\rangle}{\langle\Psi \mid \Psi\rangle}
$$

and corresponds to a free energy, as it takes into account the irreversible work spent to polarize the solvent.

In order to give a unified theoretical treatment of Hartree-Fock (HF) and Kohn-Sham density-functional theory (KS-DFT) we adopt the approach of Saue et al.77. Due to the existence of a variational principle for the nonlinear Hamiltonian $H_{\text {eff }}$, the Hohenberg-Kohn theorems of nonrelativistic DFT ${ }^{78}$ are still valid in the actual theoretical setting. Regarding the extension of DFT to a relativistic framework, we rely upon the work of Rajagopal et al. ${ }^{79}$. Throughout the derivations, we will only concentrate on the additional terms appearing due to the coupling with the polarizable continuum. Analysis of the conventional terms can be found elsewhere ${ }^{77}$.

We recall that in KS-DFT the density of the system of interest is defined to be equal to that of a noninteracting system. To this end we write the free energy as a functional of the density $\rho$ :

$$
G[\rho]=T_{s}\left[\rho_{\mathrm{e}}\right]+V_{\mathrm{ext}}[\rho]+J\left[\rho_{\mathrm{e}}\right]+E_{\mathrm{xc}}\left[\rho_{\mathrm{e}}\right]+V_{\mathrm{NN}}+\frac{1}{2} U_{\mathrm{pol}}[\rho]=E[\rho]+\frac{1}{2} U_{\mathrm{pol}}[\rho]
$$


where the final term is the polarization energy expressed as the expectation value of the $V_{\sigma \rho}[\rho]$ operator:

$$
U_{\mathrm{pol}}[\rho]=\left\langle\tilde{0}\left|V_{\sigma \rho}[\rho]\right| \tilde{0}\right\rangle=U_{\mathrm{Ne}}[\rho]+U_{\mathrm{eN}}[\rho]+U_{\mathrm{ee}}[\rho]+U_{\mathrm{NN}}
$$

In both HF and KS-DFT methods, the electron density and other quantities are obtained from a single Slater determinant built in the one-electron basis of the molecular orbitals (MOs) $\left\{\phi_{r}\right\}_{r=1, M}$. The usual notation for orbital indices will be here adopted: $i, j, k \ldots$ for occupied MOs, $a, b, c \ldots$ for virtual MOs and $p, q, r \ldots$ for general MOs. In Second Quantization a Slater determinant is represented by an Occupation Number Vector (ONV) and we will write this vector as $|0\rangle$. We choose a unitary, exponential parametrization for $|0\rangle:$

$$
|\tilde{0}\rangle=\exp (-\hat{\kappa})|0\rangle
$$

where $\hat{\kappa}$ is an anti-Hermitian operator:

$$
\hat{\kappa}=\sum_{p q} \kappa_{p q} p^{\dagger} q \quad \hat{\kappa}^{\dagger}=-\hat{\kappa}
$$

the orbital rotation coefficients thus constitute an anti-Hermitian matrix, $\boldsymbol{\kappa}^{\dagger}=-\boldsymbol{\kappa}$. The electron density can now be written as:

$$
\rho_{\mathrm{e}}(\boldsymbol{r}, \boldsymbol{\kappa})=\sum_{p q} \widetilde{D}_{p q}(\boldsymbol{\kappa}) \Omega_{p q}(\boldsymbol{r})=\sum_{p q}\left\langle 0\left|\exp (\hat{\kappa}) p^{\dagger} q \exp (-\hat{\kappa})\right| 0\right\rangle\left(\phi_{p}^{\dagger}(\boldsymbol{r}) \phi_{q}(\boldsymbol{r})\right)
$$

The advantage of this parametrization is that the orbital rotation operator $\exp (-\hat{\kappa})$ ensures orthonormality of the one-particle orbitals without the need to introduce Lagrange multipliers: unconstrained optimization approaches can be used and redundancies are easily identified. For closed-shell systems the orbital rotation operator may accordingly be 
restricted to

$$
\hat{\kappa}=\sum_{a i}\left(\kappa_{a i} a^{\dagger} i-\kappa_{a i}^{*} i^{\dagger} a\right)
$$

The gradient of the free energy with respect to the variational parameters is

$$
G_{a i}^{[1]}=\left.\frac{\partial G}{\partial \kappa_{a i}^{*}}\right|_{\kappa=0}=-f_{a i}
$$

where we have introduced the Fock (Kohn-Sham) matrix:

$$
f_{p q}=f_{p q}^{\mathrm{vac}}+j_{p q}+x_{p q}(0) ; \quad f_{p q}^{\mathrm{vac}}=h_{p q}+\sum_{j}\left(g_{p q j j}-\gamma g_{p j j q}\right)+v_{\mathrm{xc} ; p q}
$$

The xc potential $v_{\mathrm{xc} ; p q}$ depends on the actual form of the exchange-correlation functional selected (detailed expressions for LDA and GGA functionals may be found in ref. $\frac{77}{}$ ) and $\gamma$ specifies the portion of orbital exchange to be included.

Taking a variation of the form $\rho=\rho_{\mathrm{e}}+\rho_{\mathrm{N}}+\delta \rho_{\mathrm{e}}=\tilde{\rho}_{\mathrm{e}}+\rho_{\mathrm{N}}$, the polarization contributions to the Fock matrix can be derived as:

$$
\begin{aligned}
\left.\frac{1}{2} \frac{\partial U_{\mathrm{pol}}[\rho]}{\partial \kappa_{a i}^{*}}\right|_{\boldsymbol{\kappa}=\mathbf{0}} & =\left.\frac{1}{2} \int \mathrm{d} \boldsymbol{r} \frac{\delta U_{\mathrm{pol}}}{\delta \rho_{e}(\boldsymbol{r})} \frac{\partial \tilde{\rho}_{e}(\boldsymbol{r})}{\partial \kappa_{a i}^{*}}\right|_{\boldsymbol{\kappa}=\mathbf{0}}=-\int_{\mathbb{R}^{3}} \mathrm{~d} \boldsymbol{r} \int_{\mathbb{R}^{3}} \mathrm{~d} \boldsymbol{r}^{\prime} \Omega_{a i}(\boldsymbol{r}) M\left(\boldsymbol{r}, \boldsymbol{r}^{\prime}\right) \rho\left(\boldsymbol{r}^{\prime}\right) \\
& =-\int_{\partial C} \mathrm{~d} \boldsymbol{s} \int_{\partial C} \mathrm{~d} \boldsymbol{s}^{\prime} v_{a i}^{\mathrm{e}}(\boldsymbol{s}) \kappa\left(\boldsymbol{s}, \boldsymbol{s}^{\prime}\right) \phi\left(\boldsymbol{s}^{\prime}\right)=-\int_{\partial C} \mathrm{~d} \boldsymbol{s} v_{a i}^{\mathrm{e}}(\boldsymbol{s}) \sigma(\boldsymbol{s})=-\boldsymbol{q} \cdot \boldsymbol{v}_{a i}^{\mathrm{e}}
\end{aligned}
$$

where the discretization of the cavity surface was introduced in the last step. The $v_{p q, I}^{\mathrm{e}}$ integrals appearing above are given as:

$$
v_{p q, I}^{\mathrm{e}}=\int \mathrm{d} \boldsymbol{r} \frac{-\Omega_{p q}(\boldsymbol{r})}{\left|\boldsymbol{r}-\boldsymbol{s}_{I}\right|}
$$

Separation of the ASC into electronic and nuclear contributions leads to the matrix elements 
$j_{p q}$ and $x_{p q}(0)$ :

$$
\begin{aligned}
j_{p q} & =\boldsymbol{q}^{\mathrm{N}} \cdot \boldsymbol{v}_{p q}^{\mathrm{e}}=\boldsymbol{v}^{\mathrm{N \dagger}} \boldsymbol{K} \boldsymbol{v}_{p q}^{\mathrm{e}} \\
x_{p q}(0) & =\left(\sum_{t u} D_{t u} \boldsymbol{q}_{t u}^{\mathrm{e}}\right) \cdot \boldsymbol{v}_{p q}^{\mathrm{e}}=\left(\sum_{t u} D_{u t}^{*} \boldsymbol{v}_{u t}^{\mathrm{e} *}\right) \boldsymbol{K} \boldsymbol{v}_{p q}^{\mathrm{e}}
\end{aligned}
$$

The matrix elements $v_{p q, I}^{\mathrm{e}}$ in Eq. (33) shall be called the uncontracted potentials, and these are three-index quantities. A boldface notation with two indices as in $\boldsymbol{v}_{p q}^{\mathrm{e}}$ has to be interpreted as an array of dimension $N_{\text {ts }}$ whose $I$-th element is the electrostatic potential integral evaluated at the $I$-th cavity point. A scalar product of the type $\boldsymbol{q}_{p q} \cdot \boldsymbol{v}_{t u}$ is to be interpreted accordingly, i. e. as the contraction over the cavity index: $\sum_{I}^{N_{\text {ts }}} q_{p q, I} v_{t u, I}$. The stationarity condition for the electronic free energy is then simply:

$$
f_{a i}=0 \quad \forall(a i)
$$

meaning that the Fock matrix is block diagonal in the basis of the optimal solvated molecular 4-spinors and is thus equivalent to the spectral problem:

$$
F \varphi=\varphi \epsilon
$$

The derivation above applies equally well to relativistic and nonrelativistic Hamiltonians.

To gain further insight into the additional PCM contribution to the Fock (KS) matrix we may expand it in a basis of 2-spinors:

$$
\varphi_{r}=\left(\begin{array}{c}
\varphi_{r}^{\mathrm{L}} \\
\varphi_{r}^{\mathrm{S}}
\end{array}\right)=\sum_{\lambda=1}^{N_{\mathrm{Large}}}\left(\begin{array}{c}
\chi_{\lambda}^{\mathrm{L}} \\
0
\end{array}\right) C_{\lambda r}^{\mathrm{L}}+\sum_{\lambda=1}^{N_{\text {Small }}}\left(\begin{array}{c}
0 \\
\chi_{\lambda}^{\mathrm{S}}
\end{array}\right) C_{\lambda r}^{\mathrm{S}} .
$$


The form of the vacuum-like contribution to the Fock matrix is:

$$
\boldsymbol{F}^{\mathrm{vac}}=\left(\begin{array}{cc}
\boldsymbol{F}^{\mathrm{vac}, \mathrm{LL}} & \boldsymbol{F}^{\mathrm{vac}, \mathrm{LS}} \\
\boldsymbol{F}^{\mathrm{vac}, \mathrm{SL}} & \boldsymbol{F}^{\mathrm{vac}, \mathrm{SS}}
\end{array}\right)
$$

where the explicit expression of each term may be found elsewhere ${ }^{64}$.

The electrostatic potential and the corresponding polarization charge at point $I$ on the cavity is:

$$
\begin{aligned}
& v_{I}=v_{I}^{\mathrm{N}}+v_{I}^{\mathrm{e}}=\sum_{A=1}^{N_{\text {atoms }}} \frac{Z_{A}}{\left|\boldsymbol{R}_{A}-\boldsymbol{s}_{I}\right|}+\sum_{p q} D_{p q} v_{p q, I}^{\mathrm{e}} \\
& q_{I}=q_{I}^{\mathrm{N}}+q_{I}^{\mathrm{e}}=\sum_{J=1}^{N_{\mathrm{ts}}} K_{I J}\left(\sum_{A=1}^{N_{\text {atoms }}} \frac{Z_{A}}{\left|\boldsymbol{R}_{A}-\boldsymbol{s}_{J}\right|}+\sum_{p q} D_{p q} v_{p q, J}^{\mathrm{e}}\right)
\end{aligned}
$$

and we can expand $v_{I}^{\mathrm{e}}$ in our 2-spinor basis as:

$$
v_{I}^{\mathrm{e}}=\sum_{p q} D_{p q} v_{p q, I}^{\mathrm{e}}=\sum_{p q} \sum_{X Y} \sum_{\kappa \lambda} C_{\kappa p}^{\mathrm{X} *} D_{p q} C_{\lambda q}^{\mathrm{Y}} v_{\kappa \lambda, I}^{\mathrm{e}, \mathrm{XY}}
$$

we now define the density matrices in a 2-spinor basis as:

$$
D_{\mu \nu}^{X Y}=\sum_{p q} C_{\nu p}^{\mathrm{X} *} D_{p q} C_{\mu q}^{\mathrm{Y}}=\sum_{i} C_{\mu i}^{\mathrm{Y}} C_{\nu i}^{\mathrm{X} *}
$$

the last equality being valid only for a closed-shell SCF wave function. We note that the $v_{\kappa \lambda, I}^{\mathrm{e}, \mathrm{XY}}$ are calculated over a purely multiplicative Coulomb interaction kernel that is an even operator and hence do not couple the large and small components of 4-spinors:

$$
v_{\kappa \lambda, I}^{\mathrm{e}, \mathrm{XY}}=\int \mathrm{d} \boldsymbol{r} \chi_{\kappa}^{X \dagger}(\boldsymbol{r}) \frac{-1}{\left|\boldsymbol{r}-\boldsymbol{s}_{I}\right|} \chi_{\lambda}^{Y}(\boldsymbol{r})=\delta_{X Y} \int \mathrm{d} \boldsymbol{r} \frac{-\Omega_{\kappa \lambda}^{X Y}(\boldsymbol{r})}{\left|\boldsymbol{r}-\boldsymbol{s}_{I}\right|}
$$

Eq. 39a for the potential and consequently Eq. (39b) are simplified, as their matrix 
representation is block diagonal in a 2-spinor basis:

$$
\begin{aligned}
& v_{I}=v_{I}^{\mathrm{N}}+v_{I}^{\mathrm{e}, \mathrm{LL}}+v_{I}^{\mathrm{e}, \mathrm{SS}} \\
& q_{I}=q_{I}^{\mathrm{N}}+q_{I}^{\mathrm{e}, \mathrm{LL}}+q_{I}^{\mathrm{e}, \mathrm{SS}}
\end{aligned}
$$

Inserting the result in Eq. (42) in Eqs. (34a) and (34b) the 2-spinor expansion of the $J$ and $X(0)$ solvent operators is obtained:

$$
j_{\kappa \lambda}^{X X}=\boldsymbol{q}^{\mathrm{N}} \cdot \boldsymbol{v}_{\kappa \lambda}^{\mathrm{e}, \mathrm{XX}} \quad x_{\kappa \lambda}^{X X}(0)=\boldsymbol{q}^{\mathrm{e}} \cdot \boldsymbol{v}_{\kappa \lambda}^{\mathrm{e}, \mathrm{XX}}
$$

To conclude, the final form of the Fock matrix in solution is:

$$
\boldsymbol{F}=\left(\begin{array}{cc}
\boldsymbol{F}^{\mathrm{vac}, \mathrm{LL}}+\boldsymbol{q} \cdot \boldsymbol{v}^{\mathrm{e}, \mathrm{LL}} & \boldsymbol{F}^{\mathrm{vac}, \mathrm{LS}} \\
\boldsymbol{F}^{\mathrm{vac}, \mathrm{SL}} & \boldsymbol{F}^{\mathrm{vac}, \mathrm{SS}}+\boldsymbol{q} \cdot \boldsymbol{v}^{\mathrm{e}, \mathrm{SS}}
\end{array}\right)
$$

where the PCM contribution appears only on its diagonal blocks.

Let us now consider possible approximations of the polarization contribution in order to achieve computational speedups. It is well known that the most intensive task in an electronic structure calculation is the construction of matrix elements involving two-electron integrals. This computational bottleneck is even worse for calculations based on the Dirac-Coulomb Hamiltonian since the number of atomic basis functions is always greater than the one in a nonrelativistic calculation on the same system due to the small components of the molecular 4-spinor. However, due to the locality of the small component density, the calculation of two-electron integrals of the $g^{\mathrm{SSSS}}$ class can be completely avoided $\underline{81}$. Neglecting the very expensive $g^{\text {SSSS }}$ class of two-electron integrals and applying an a posteriori simple Coulombic correction (SCC) to the energy with pretabulated or computed small component charges leads to a negligible error in energies, structures and molecular observables.

We propose a similar approximation, called PCM-SCC, to avoid the calculation of the 
$\boldsymbol{v}^{\mathrm{SS}}$ class of integrals. The electronic molecular electrostatic potential at cavity point $I$ is:

$$
v_{I}^{\mathrm{e}}=\sum_{\kappa \lambda} D_{\kappa \lambda}^{\mathrm{LL}} v_{\lambda \kappa, I}^{\mathrm{e}, L L}+\sum_{\kappa \lambda} D_{\kappa \lambda}^{\mathrm{SS}} v_{\lambda \kappa, I}^{\mathrm{e}, S S} \simeq \sum_{\kappa \lambda} D_{\kappa \lambda}^{\mathrm{LL}} v_{\lambda \kappa, I}^{\mathrm{e}, L L}+\sum_{A}^{N_{\text {nuclei }}} \frac{q_{A}^{\mathrm{SCC}}}{\left|\boldsymbol{R}_{A}-\boldsymbol{s}_{I}\right|}
$$

where $q_{A}^{\mathrm{SCC}}$ is the pretabulated small charge for nucleus $A$. Finally, in the spirit of the SCC

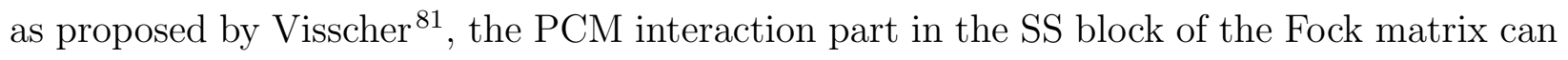
be completely neglected:

$$
\boldsymbol{F}=\left(\begin{array}{cc}
\boldsymbol{F}^{\mathrm{vac}, \mathrm{LL}}+\boldsymbol{q} \cdot \boldsymbol{v}^{\mathrm{e}, \mathrm{LL}} & \boldsymbol{F}^{\mathrm{vac}, \mathrm{LS}} \\
\boldsymbol{F}^{\mathrm{vac}, \mathrm{SL}} & \boldsymbol{F}^{\mathrm{vac}, \mathrm{SS}}+\boldsymbol{q} \cdot \boldsymbol{v}^{\mathrm{e}, \mathrm{SS}}
\end{array}\right) \simeq\left(\begin{array}{cc}
\boldsymbol{F}^{\mathrm{vac}, \mathrm{LL}}+\boldsymbol{q} \cdot \boldsymbol{v}^{\mathrm{e}, \mathrm{LL}} & \boldsymbol{F}^{\mathrm{vac}, \mathrm{LS}} \\
\boldsymbol{F}^{\mathrm{vac}, \mathrm{SL}} & \boldsymbol{F}^{\mathrm{vac}, \mathrm{SS}}
\end{array}\right)
$$

The reader may wonder why one has to bother with such approximate schemes when the molecular electrostatic potential matrix elements are one-electron integrals, usually quite cheap to compute. One must however bear in mind that such integrals are to be calculated over the grid points provided by the discretization of the cavity. This implies that the formal scaling of the PCM contributions is on the order of $N_{\mathrm{ts}} N^{2}$ compared to $N^{4}$ for the construction of the two-electron Fock matrix. The number of tesserae $N_{\mathrm{ts}}$ depends on the molecular topology and the user-specified average tesserae area $a_{I}$ and is independent of the number $N$ of basis functions. If reduced scaling algorithms are applied to the two-electron part such algorithms must also be applied to the PCM contribution to avoid that the latter becomes a computational bottleneck.

\subsection{Linear response for a relativistic PCM-SCF state}

In order to derive the linear response function for static perturbations, we augment the free energy functional in Eq. 23) with a perturbation operator $V$ :

$$
G[0]=\left\langle 0\left|H_{0}+\frac{1}{2} V_{\sigma \rho}[\rho]+V\right| 0\right\rangle
$$


The perturbation operator is taken to have the following form:

$$
V=\sum_{X} \epsilon_{X} H_{X}
$$

i.e. a linear combination of one-electron perturbation operators $H_{X}$ weighted by the perturbation strengths $\epsilon_{X}$. Molecular properties are then obtained as derivatives, at zero perturbation strength, of the free energy with respect to the perturbation strength. We will closely follow Sałek et al. .82 , but focus on solvent contributions.

Notice that the free energy functional in Eq. (48) does not take into account nonequilibrium effects 83 . It is thus not suitable for the derivation of frequency-dependent response functions, needed for the calculation of dynamic properties and excitation energies 84 . We shall address the extension of the current derivation to the nonequilibrium regime in a later contribution.

Using the variational condition (35), second-order properties can be expressed as

$$
\left.\frac{\mathrm{d}^{2} G}{\mathrm{~d} \epsilon_{A} \mathrm{~d} \epsilon_{B}}\right|_{\epsilon=0}=\left.\sum_{p q} \frac{\partial^{2} G}{\partial \epsilon_{A} \partial \kappa_{p q}} \frac{\mathrm{d} \kappa_{p q}}{\mathrm{~d} \epsilon_{B}}\right|_{\epsilon=0} .
$$

The first-order amplitudes are obtained from the first-order response equation

$$
\left.\frac{\mathrm{d}}{\mathrm{d} \epsilon_{B}}\left(\frac{\partial G}{\partial \kappa_{p q}}\right)\right|_{\epsilon=0}=\left[\frac{\partial^{2} G}{\partial \epsilon_{B} \partial \kappa_{p q}}+\sum_{r s} \frac{\partial^{2} G}{\partial \kappa_{p q} \partial \kappa_{r s}} \frac{\mathrm{d} \kappa_{r s}}{\mathrm{~d} \epsilon_{B}}\right]_{\epsilon=0}=0
$$

which can be recast in matrix form as

$$
\boldsymbol{G}^{[2]} \boldsymbol{X}_{B}=-\boldsymbol{E}_{B}^{[1]}
$$

The electronic free energy Hessian appearing in this equation has the following structure ${ }^{82}$ :

$$
\boldsymbol{G}^{[2]}=\left(\begin{array}{cc}
\boldsymbol{A} & \boldsymbol{B} \\
\boldsymbol{B}^{*} & \boldsymbol{A}^{*}
\end{array}\right)
$$


with the $\boldsymbol{A}$ and $\boldsymbol{B}$ matrix elements being:

$$
\begin{aligned}
& \left.\frac{\partial^{2} G}{\partial \kappa_{a i}^{*} \partial \kappa_{b j}}\right|_{\kappa=0}=A_{a i, b j}=\delta_{i j} f_{a b}-\delta_{a b} f_{j i}+L_{a i, j b}^{\gamma}+w_{\mathrm{xc} ; a i, j b}+\boldsymbol{q}_{a i}^{\mathrm{e}} \cdot \boldsymbol{v}_{j b}^{\mathrm{e}} \\
& \left.\frac{\partial^{2} G}{\partial \kappa_{a i}^{*} \partial \kappa_{b j}^{*}}\right|_{\kappa=0}=B_{a i, b j}=L_{a i, b j}^{\gamma}+w_{\mathrm{xc} ; a i, b j}+\boldsymbol{q}_{a i}^{\mathrm{e}} \cdot \boldsymbol{v}_{b j}^{\mathrm{e}}
\end{aligned}
$$

where

$$
f_{p q}=f_{p q}^{\mathrm{vac}}+\boldsymbol{q} \cdot \boldsymbol{v}_{p q}^{\mathrm{e}} \quad L_{a i, j b}^{\gamma}=g_{a i j b}-\gamma g_{a b j i} .
$$

The vector of apparent surface charges is given as $\boldsymbol{q}=\boldsymbol{q}^{\mathrm{N}}+\boldsymbol{q}^{\mathrm{e}}$. The solvent contributions to the $\boldsymbol{A}$ matrix are found as

$$
\begin{aligned}
A_{a i, b j}^{\mathrm{pol}} & =\left.\frac{1}{2} \int \mathrm{d} \boldsymbol{r} \frac{\delta U_{\mathrm{pol}}}{\delta \rho_{\mathrm{e}}(\boldsymbol{r})} \frac{\partial^{2} \rho_{\mathrm{e}}(\boldsymbol{r})}{\partial \kappa_{a i}^{*} \partial \kappa_{b j}}\right|_{\boldsymbol{\kappa}=\mathbf{0}}+\left.\int_{\mathbb{R}^{3}} \mathrm{~d} \boldsymbol{r} \int_{\mathbb{R}^{3}} \mathrm{~d} \boldsymbol{r}^{\prime} \frac{\delta^{2} U_{p o l}}{\delta \rho_{e}(\boldsymbol{r}) \delta \rho_{e}\left(\boldsymbol{r}^{\prime}\right)} \frac{\partial \rho_{\mathrm{e}}(\boldsymbol{r})}{\partial \kappa_{a i}^{*}} \frac{\partial \rho_{\mathrm{e}}\left(\boldsymbol{r}^{\prime}\right)}{\partial \kappa_{b j}}\right|_{\boldsymbol{\kappa}=\mathbf{0}} \\
& =\left.\frac{1}{2} \int \mathrm{d} \boldsymbol{r} \frac{\delta U_{\mathrm{pol}}}{\delta \rho_{\mathrm{e}}(\boldsymbol{r})}\left[\delta_{i j} \Omega_{a b}(\boldsymbol{r})-\delta_{a b} \Omega_{j i}(\boldsymbol{r})\right]\right|_{\boldsymbol{\kappa}=\mathbf{0}}+\left.\int_{\mathbb{R}^{3}} \mathrm{~d} \boldsymbol{r} \int_{\mathbb{R}^{3}} \mathrm{~d} \boldsymbol{r}^{\prime} \frac{\delta^{2} U_{\mathrm{pol}}}{\delta \rho_{\mathrm{e}}(\boldsymbol{r}) \delta \rho_{\mathrm{e}}\left(\boldsymbol{r}^{\prime}\right)} \Omega_{a i}(\boldsymbol{r}) \Omega_{j b}\left(\boldsymbol{r}^{\prime}\right)\right|_{\boldsymbol{\kappa}=\mathbf{0}} \\
& =\delta_{i j}\left(\boldsymbol{q} \cdot \boldsymbol{v}_{a b}^{\mathrm{e}}\right)-\delta_{a b}\left(\boldsymbol{q} \cdot \boldsymbol{v}_{j i}^{\mathrm{e}}\right)+\boldsymbol{q}_{a i}^{\mathrm{e}} \cdot \boldsymbol{v}_{j b}^{\mathrm{e}}
\end{aligned}
$$

and analogously for the $\boldsymbol{B}$ matrix.

The solution of the linear system in Eq. (52) is achieved by means of subspace iteration methods ${ }^{85}$. The solution vector is expanded in a set of $n$ trial vectors, $\boldsymbol{b}_{i}$, leading to the reduced response equation, i. e. their projection in the chosen subspace. Such a method requires repeated evaluation of the so-called $\boldsymbol{\sigma}$-vector, i. e. the linear transformation of the selected subspace by the electronic free energy Hessian.

The $\boldsymbol{\sigma}$-vector formation can be reformulated as the evaluation of a generalized Fock matrix 86 :

$$
\sigma_{a i}=-\left[\widetilde{f}_{a i}+L_{a i}^{\gamma}+\widetilde{\boldsymbol{q}}^{\mathrm{e}} \cdot \boldsymbol{v}_{a i}^{\mathrm{e}}\right]
$$

In these expressions a tilde indicates that a one-index transformation of the integrals by 
means of the trial vector has to be performed. The expression of the transformed two-

electron term $L_{r s}^{\gamma}$ is given elsewhere ${ }^{86 \mid 87}$. What is to be noted here is that it can be evaluated by contraction of the usual two electron integrals with a perturbed AO basis one-electron density, obtained by transformation with the trial vectors. A similar approach can be used in evaluating the one-index transformed polarization charges:

$$
\widetilde{\boldsymbol{q}}^{\mathrm{e}}=\boldsymbol{K}\left[\sum_{\kappa \lambda} \widetilde{D}_{\kappa \lambda} \boldsymbol{v}_{\lambda \kappa}^{\mathrm{e}}\right] \quad \widetilde{D}_{\kappa \lambda}=-\sum_{u t} C_{u \lambda} b_{u t} C_{t \kappa}^{*}
$$

\section{Implementation}

The solution of the electrostatic problem posed by the addition of a polarizable continuum surrounding the molecular solute, requires a limited number of steps which are independent of the nature of the electronic-structure method employed. These steps, namely, cavity formation and discretization together with formation of the PCM matrix $\boldsymbol{K}$, can be abstracted from the structure of the program performing the optimization of the electronic structure. This modular programming paradigm is not new $\underline{88 \mid 89}$, but is a very powerful strategy to effectively enable code reuse throughout altogether different quantum chemical programs.

In Figure 1 we show the PCM-SCF algorithm one needs to implement. The neat separation between PCM-related and QM-related tasks is shown, the only additional step added with respect to a conventional in vacuo SCF algorithm being the evaluation of the molecular electrostatic potential at the grid points provided by the discretized molecular cavity.

The existence of this separation between the classical electrostatic problem and the quantum problem for the optimization of the electronic structure, led us to the implementation of a standalone module for the PCM, which we have called PCMSolver 61. PCMSolver is intended to be an application programming interface (API) providing all the functionality needed to handle the PCM electrostatic problem: generation and discretization of the cavity, generation of the PCM matrix. Both tasks can be performed in a fully general manner: 


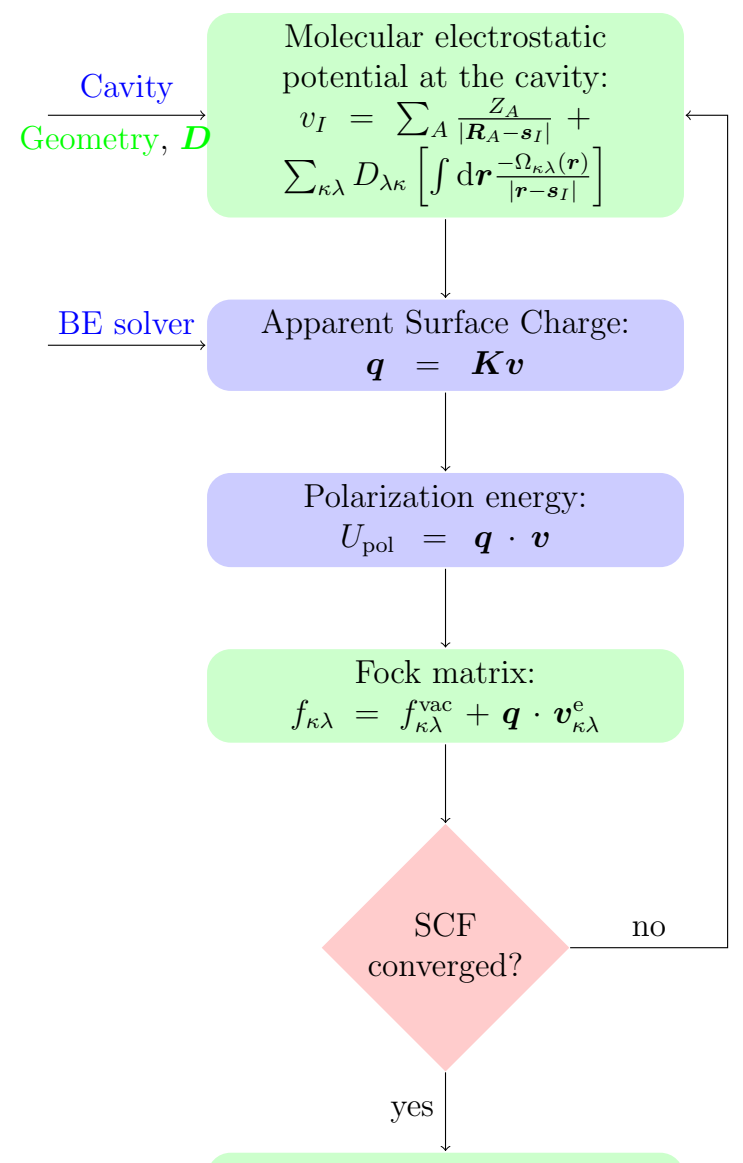

Finalize SCF

Figure 1: Schematic view of the implemented SCF algorithm. Computations/data in blue are on the PCMSolver side, in green on the DIRAC side.

isotropic and anisotropic environments can be treated, and are accommodated within the same general code infrastructure, thus reflecting the derivation of the IEF-PCM equation given by Cancès et al. ${ }^{72}$ in their seminal paper. Treatment of diffuse interfaces is also possible ${ }^{\sqrt{90}}$. The newly implemented Boundary Element solver based on a wavelet formalism is made available within the same framework ${ }^{91}[93$.

The concept of data hiding is effectively enforced: only the necessary functions are visible to the end-user of our API through an interface. The major coding effort in interfacing PCMSolver to any quantum chemical program regards the efficient evaluation of the molecular electrostatic potential. We would like to stress the point that through the use of our API, virtually any quantum chemical program package could introduce a continuum description 
of the solvent. Our approach has two main advantages over an in-house coding of the PCM:

1. coding effort is minimized, because the necessary functions already come bundled in a compact library. Furthermore, these functions are already tested, only the QM-PCM interface is to be tested;

2. new PCM functionalities, such as novel algorithms for the cavity generation and the solution of the electrostatic problem, as well as additional environments, can be added to the API without touching the QM code. These new functionalities will be seamlessly and immediately available to the QM program, with a negligible amount of work.

\subsection{4-component Molecular Electrostatic Potential}

As shown in Section 2.1, the addition of relativity is irrelevant for the generic algorithm which is suitable for both nonrelativistic and relativistic calculations. The only difference in the latter case is in the calculation of the contracted electrostatic potential

$$
v_{I}^{\mathrm{e}}=\sum_{p q} D_{p q} v_{p q, I}^{\mathrm{e}},
$$

In a scalar atomic basis, we need to calculate the integrals

$$
v_{\kappa \lambda}^{I}\left(\boldsymbol{s}_{I}\right)=\int \mathrm{d} \boldsymbol{r} \frac{-\Omega_{\kappa \lambda}(\boldsymbol{r})}{\left|\boldsymbol{r}-\boldsymbol{s}_{I}\right|} .
$$

where $\boldsymbol{s}_{I}$ is a point on the cavity surface. Such integrals are identical to the ordinary nuclearattraction integrals, but have a different physical origin and should rather be called chargeattraction integrals. We recall that only the LL and SS sub-blocks need to be evaluated.

The implementation of Eq. (60) requires looping over basis functions and grid points. The loop over grid points may be placed either outside or inside the two basis function loops. The former choice is easier to implement but generates a highly inefficient code, due to the large number of intermediate quantities that needs to be recalculated for each grid 
point. The latter is instead more efficient because it can be seen as a form of vectorization, where each iteration over the basis function, an entire batch of points (the whole grid in our case), is computed instead of one point at a time. Intermediates are in this case reused and a full exploitation of compiler optimization is possible. The vectorization will also enable a relatively straightforward port of the code to architectures based on General-purpose computing on graphics processing units (GPGPU).

This second approach is the one used in our implementation in the DIRAC code ${ }^{60}$. As a useful by-product, molecular electrostatic potential maps are available for 4-component electronic-structure calculations. To the best of our knowledge, this is the first implementation of such a visualization and analysis tool in a relativistic 4-component framework.

\section{Applications}

As a first application we have considered the geometries, electric dipole moments and static electric dipole polarizabilities for the series of the group 16 dihydrides, $\mathrm{H}_{2} \mathrm{O}$ through $\mathrm{H}_{2} \mathrm{Po}$, both in vacuo and in water. To the best of our knowledge, no experimental values for the observables here considered are available for $\mathrm{H}_{2} \mathrm{Te}$ and $\mathrm{H}_{2} \mathrm{Po}$. A limited number of theoretical results is available ${ }^{94101}$ for the same species in vacuo. In the following, no attempt is made to compare our results with the ones reported in the literature since our results are only to be taken as an illustration of the reported implementation.

\subsection{Computational methods}

All calculations were performed at the Hartree-Fock and DFT levels of theory, the latter using the hybrid-GGA exchange-correlation functional PBE0 $0^{102}$. 4-component relativistic calculations are based on the Dirac-Coulomb Hamiltonian, with or without ${ }^{103}$ spin-orbit interaction, whereas non-relativistic calculations are based on the 4-component Lévy-Leblond Hamiltonian. ${ }^{71}$ The $g^{\text {SSSS }}$ class of two-electron integrals was neglected in all calculations and 


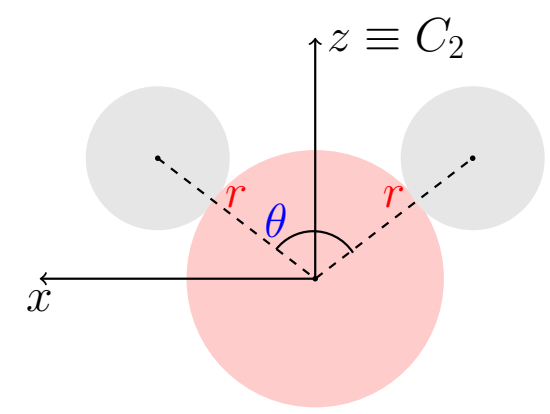

Figure 2: Geometric parameters and orientation of the $\mathrm{H}_{2} \mathrm{X}$ species considered. The positive $y$ axis points outside the $x z$-plane.

Visscher's simple Coulombic correction ${ }^{81}$ adopted throughout.

A development version of the 4-component relativistic molecular code DIRAC, interfaced with the PCMSolver module, was used. Uncontracted, triple-zeta quality basis sets were used for the large components: cc-pVTZ for H, O, $\mathrm{S}^{104 \mid 105}$ and dyall.v3z ${ }^{106}[108$ for Se, Te, Po. Restricted kinetic balance was applied to obtain the small component basis set.

In all calculations, the dihydrides are placed in the $x z$-plane, the $C_{2}$ rotation axis is along the $z$ axis, with the direction of the positive $z$ axis from the heavy atom to the hydrogens, as shown in Figure 2.

The structures were optimized using the numerical molecular gradient evaluated by means of finite differences. The general driver for structure optimizations in DIRAC is the same as in the nonrelativistic code DALTON $109 \mid 110$. The structures optimized in vacuo using the numerical gradient were compared with those obtained using the analytic gradient. Bond lengths, bond angles and energies obtained with the two methods were found to be in good agreement: for Hartree-Fock calculations, the average relative error in bond lengths and bond angles is 80 ppm, while that on energies is 50 ppb. Based on these results, we have assumed the numerical gradient optimizations to be reliable also for solvent calculations, where an analytic gradient is not yet available.

Water, with relative dielectric constant $\varepsilon_{r}=78.39$, was selected as solvent. The cavities were generated using the Bondi-Mantina set of van der Waals radii 111 . The radii used 1.20 $\AA$ for $\mathrm{H}, 1.52 \AA$ for $\mathrm{O}, 1.80 \AA$ for $\mathrm{S}, 1.90 \AA$ for Se, $2.06 \AA$ for $\mathrm{Te}$ and $1.97 \AA$ for Po. These 
radii were then multiplied by a scaling factor of 1.2 as usual in the application of the PCM 112 . The cavities were obtained without the addition of spheres not centered on the nuclei. A fine tessellation, with average tessera area of $0.3 a_{0}^{2} \simeq 0.084 \AA^{2}$, was chosen. For $\mathrm{H}_{2} \mathrm{O}$ and $\mathrm{H}_{2} \mathrm{Po}$, we investigated the effect of tessellation (not shown) and found our results converged for this value of the average tessera area, as could have been expected from non-relativistic studies. For further discussion of tessellation and other technical issues related to the implementation

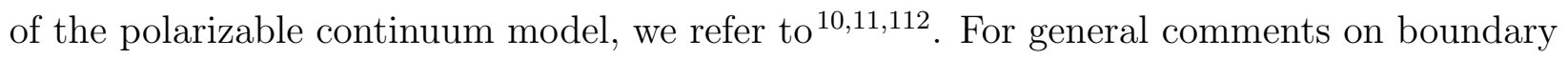
element methods for integral equations, such as discretization techniques and convergence estimates, we refer to the book by Hackbusch ${ }^{74}$.

For electronic structure analysis we have employed projection analysis ${ }^{100}$ using the precalculated in vacuo orbitals of the constituent atoms.

\subsection{Assessment of the PCM-SCC approximation}

The possibility to skip the evaluation of the SS block of the electrostatic potential integrals as described in Section 2.3, was implemented (.SKIPSS input keyword). By default, these integrals are not skipped. To assess the impact of the PCM-SCC approximation, geometry optimizations were performed for $\mathrm{H}_{2} \mathrm{Po}$, both at the Hartree-Fock and DFT/PBE0 levels of theory. Subsequent single-point calculations employing the "full" Dirac-Coulomb model and the approximate PCM-SCC model were performed, taking the geometry optimized with the "full" model as reference.

Tables 1 and 2 summarize the results obtained for geometries, energies and CPU times. All calculations were performed on a single node equipped with two Xeon E5-2670@2.60 GHz octacore processors. The MPI-parallel version of the code was used. The geometries predicted with the proposed approximation to the electrostatic potential integrals fully agree with the ones obtained with the "full" model. The agreement between calculated energies is also found to be acceptable. Although the formation of the PCM contribution to the Fock matrix was not found to be the most time consuming step in our test case, the timings 
reported in Table 2 suggests that it may be beneficial to employ the PCM-SCC approximation in those cases where one or both of the following conditions apply: a) a large number of small component basis functions is used; b) a large number of finite elements is used to discretize the PCM cavity. All the calculations presented in the rest of this work were performed without resorting to the PCM-SCC approximation.

Table 1: Differences in bond distance, bond angle and free energy between the Dirac-Coulomb and Dirac-Coulomb PCM-SCC results for $\mathbf{H}_{2}$ Po. Single-point calculations performed on the geometry optimized with the Dirac-Coulomb Hamiltonian.

\begin{tabular}{cccc}
\hline & $\Delta r / \AA$ & $\Delta \theta /{ }^{\circ}$ & $\Delta G / E_{\mathrm{h}}$ \\
\hline Hartree-Fock & -0.00001 & 0.002 & 0.000003 \\
DFT/PBE0 & -0.00002 & -0.001 & 0.000007 \\
\hline
\end{tabular}

Table 2: Average CPU time elapsed in an SCF iteration, $t_{\mathrm{SCF}}$, and in the formation of the PCM contribution to the Fock matrix, $t_{\mathrm{PCM}}$. The number of iterations needed to reach convergence, $N_{\text {it }}$ is also reported. All timings in seconds, calculations performed on a single node equipped with two Xeon E5-2670@2.60 GHz octacore processors. The system studied was $\mathrm{H}_{2} \mathrm{Po}$.

\begin{tabular}{lcccccc}
\hline & \multicolumn{3}{c}{ Dirac-Coulomb } & \multicolumn{3}{c}{ Dirac-Coulomb PCM-SCC } \\
\cline { 2 - 7 } & $t_{\mathrm{SCF}}$ & $t_{\mathrm{PCM}}$ & $N_{\text {it }}$ & $t_{\mathrm{SCF}}$ & $t_{\mathrm{PCM}}$ & $N_{\text {it }}$ \\
\hline Hartree-Fock & 43.16 & 4.90 & 27 & 35.47 & 0.86 & 21 \\
DFT/PBE0 & 47.62 & 5.40 & 19 & 38.22 & 0.90 & 18 \\
\hline
\end{tabular}

As illustrated in Figure 1, in each SCF cycle we perform the following steps:

1. we form the uncontracted potentials Eq. (33) and immediately contract them with the density matrix, to obtain the potential at cavity points. Here we have a saving since the SS block is neglected and approximated with a SCC-like correction;

2. the polarization charges are calculated. This is a call to PCMSolver that performs a matrix-vector multiplication. There is no saving here as this depends solely on the dimension of the cavity which is unaffected by skipping the SS block; 
3. we again form the uncontracted potentials Eq. (33) and immediately contract them with the polarization charges, to obtain the PCM contribution to the Fock matrix. Here we have a saving since there is no SS block, see Eq. (47);

The reader may notice that in each SCF cycle, there is an additional time saving, not accounted for by the savings in $t_{\mathrm{PCM}}$. This is because in each SCF step the PCM-SCC approximation has an impact both in the initial formation of the potential at cavity points and in the formation of the PCM contribution to the Fock matrix.

\subsection{Relativistic effects associated with the PCM model}

Key parameters in the PCM model are the atomic radii used for the generation of the molecular cavity. As already stated we have in the present work employed the Bondi-Mantina set of van der Waals radii ${ }^{[111}$ scaled by a factor of 1.2 , consistent with previous PCM calculations. ${ }^{112}$ Whereas Bondi extracted his recommended van der Waals radii from contact distances from X-ray diffraction studies of (mostly) molecular crystals, $\frac{113}{{ }^{113}}$ Mantina et al. extended the tables by calculations of the repulsive wall distance with respect to neutral, closed-shell probes.111 The radii for the heavier elements were obtained using the Douglas-Kroll-Hess Hamiltonian including scalar relativistic effects only. It would be worth investigating the trend in van der Waals radii when considering more complete Hamiltonian models, in particular for the heavier $p$-block elements where second-order spin-orbit effects, affecting orbital sizes, are substantial, but this is outside the scope of the present contribution.

It should also be noted that the apparent surface charges in the PCM model will induce a spin-orbit effect in addition to those generated by the relative motion of a reference electron and other charges, electrons and nuclei, in the system. ${ }^{63}$ This effect is difficult to quantify, but we observe that for $\mathrm{H}_{2} \mathrm{Po}$, at the optimized DC/PBE0 geometry in water, the magnitude of the solvation energy is reduced by $\simeq 15 \%$ when the spin-orbit interaction is turned off, albeit most of this effect probably arises from the modification of the electron density. 


\subsection{Geometries and electric dipole moments}

Table 3 presents the results regarding the geometries and electric dipole moments obtained at the Hartree-Fock and DFT/PBE0 levels of theory. Only the $z$-component of the electric dipole moment is reported, since the $x$ - and $y$-components are zero by symmetry.

We recall that the $I$-th component of the electric dipole moment is defined as the first derivative of the (free) energy with respect to the $I$-th component of an applied electric field $F^{114}$, which is equivalent, in the case of SCF methods, to the calculation of the expectation value of the electric dipole operator:

$$
\mu_{I}=-\left.\frac{\partial E(\boldsymbol{F})}{\partial F_{I}}\right|_{\boldsymbol{F}=0}=\left\langle 0\left|\hat{\mu}_{I}\right| 0\right\rangle
$$

Table 3: Bond lengths, bond angles and z-components of the electric dipole moment.

\begin{tabular}{|c|c|c|c|c|c|c|c|c|c|c|c|}
\hline & & & \multicolumn{3}{|c|}{ Dirac-Coulomb } & \multicolumn{3}{|c|}{ Spin-free } & \multicolumn{3}{|c|}{ Lévy-Leblond } \\
\hline & & & $r / \AA$ & $\theta / \circ$ & $\mu_{z} / \mathrm{D}$ & $r / \AA$ & $\theta / \circ$ & $\mu_{z} / \mathrm{D}$ & $r / \AA$ & $\theta / \circ$ & $\mu_{z} / \mathrm{D}$ \\
\hline \multirow{10}{*}{ Hartree-Fock } & \multirow{5}{*}{ In vacuo } & $\mathrm{H}_{2} \mathrm{O}$ & 0.940 & 105.9 & 1.985 & 0.940 & 105.9 & 1.985 & 0.941 & 106.0 & 1.988 \\
\hline & & $\mathrm{H}_{2} \mathrm{~S}$ & 1.329 & 94.1 & 1.142 & 1.329 & 94.1 & 1.143 & 1.330 & 94.2 & 1.160 \\
\hline & & $\mathrm{H}_{2}{ }_{2} \mathrm{Se}$ & 1.451 & 92.9 & 0.779 & 1.451 & 92.9 & 0.782 & 1.454 & 93.2 & 0.858 \\
\hline & & $\mathrm{H}_{2} \mathrm{Te}$ & 1.649 & 92.1 & 0.293 & 1.648 & 92.2 & 0.312 & 1.656 & 92.7 & 0.495 \\
\hline & & $\mathrm{H}_{2}^{2} \mathrm{Po}$ & 1.742 & 90.8 & -0.575 & 1.725 & 91.2 & -0.311 & 1.754 & 92.6 & 0.261 \\
\hline & \multirow{5}{*}{ Water } & $\mathrm{H}_{2} \mathrm{O}$ & 0.944 & 104.9 & 2.296 & 0.944 & 104.9 & 2.296 & 0.944 & 104.9 & 2.299 \\
\hline & & $\mathrm{H}_{2} \mathrm{~S}$ & 1.331 & 94.9 & 1.484 & 1.331 & 94.9 & 1.484 & 1.331 & 95.0 & 1.505 \\
\hline & & $\mathrm{H}_{2} \mathrm{Se}$ & 1.452 & 93.7 & 1.082 & 1.452 & 93.7 & 1.085 & 1.455 & 94.0 & 1.182 \\
\hline & & $\mathrm{H}_{2} \mathrm{Te}$ & 1.649 & 92.6 & 0.485 & 1.648 & 92.6 & 0.508 & 1.657 & 93.4 & 0.758 \\
\hline & & $\mathrm{H}_{2} \mathrm{Po}$ & 1.746 & 90.0 & -0.832 & 1.728 & 90.7 & -0.444 & 1.754 & 92.7 & 0.398 \\
\hline \multirow{10}{*}{ DFT/PBE0 } & \multirow{5}{*}{ In vacuo } & $\mathrm{H}_{2} \mathrm{O}$ & 0.958 & 104.3 & 1.923 & 0.958 & 104.3 & 1.923 & 0.958 & 104.4 & 1.926 \\
\hline & & $\mathrm{H}_{2} \mathrm{~S}$ & 1.344 & 92.2 & 1.118 & 1.344 & 92.2 & 1.118 & 1.344 & 92.3 & 1.136 \\
\hline & & $\mathrm{H}_{2}{ }_{2} \mathrm{Se}$ & 1.465 & 90.8 & 0.719 & 1.465 & 90.8 & 0.722 & 1.467 & 91.1 & 0.801 \\
\hline & & $\mathrm{H}_{2} \mathrm{Te}$ & 1.661 & 90.2 & 0.239 & 1.660 & 90.2 & 0.259 & 1.667 & 90.8 & 0.447 \\
\hline & & $\mathrm{H}_{2} \mathrm{Po}$ & 1.759 & 89.3 & -0.577 & 1.738 & 89.7 & -0.324 & 1.762 & 90.8 & 0.233 \\
\hline & \multirow{5}{*}{ Water } & $\mathrm{H}_{2} \mathrm{O}$ & 0.962 & 103.3 & 2.249 & 0.962 & 103.3 & 2.249 & 0.962 & 103.4 & 2.252 \\
\hline & & $\mathrm{H}_{2} \mathrm{~S}$ & 1.346 & 93.1 & 1.466 & 1.346 & 93.1 & 1.466 & 1.346 & 93.2 & 1.488 \\
\hline & & $\mathrm{H}_{2} \mathrm{Se}$ & 1.467 & 91.6 & 1.009 & 1.466 & 91.6 & 1.012 & 1.469 & 92.0 & 1.114 \\
\hline & & $\mathrm{H}_{2} \mathrm{Te}$ & 1.662 & 90.7 & 0.409 & 1.661 & 90.7 & 0.433 & 1.668 & 91.5 & 0.694 \\
\hline & & $\mathrm{H}_{2} \mathrm{Po}$ & 1.764 & 88.9 & -0.842 & 1.741 & 89.1 & -0.477 & 1.763 & 91.0 & 0.351 \\
\hline
\end{tabular}

Trends in bond lengths and bond angles along the periods are reported in Figure 3 and 
Figure 4, respectively. The geometries predicted at the Hartree-Fock level of theory show shorter bond lengths and larger bond angles than the ones predicted using DFT/PBE0. A monotonic increase in bond length, correlating with the size of the central atom, is observed going down in the group. No deviations from this trend are observed, neither including relativity, nor considering the solvent. Scalar relativistic effects tend, as expected, to shorten bonds. Spin-orbit effects only become dramatic for $\mathrm{H}_{2} \mathrm{Po}$ : At the DFT/PBE0/in vacuo level of theory scalar relativity shortens the bond by $0.024 \AA$, whereas spin-orbit interaction increases the bond length by $0.021 \AA$, almost back to the non-relativistic value.

Figure 4 clearly shows a marked reduction in bond angle beyond water, which constitutes a well-known failure of the valence shell electron pair repulsion (VSEPR) model. These observations agree with the study by Dubillard et al. 100, where a detailed discussion is provided. The effect of the solvent on molecular geometries is seen to be rather small: bond lengths increase slightly, whereas there is no clear trend for bond angles.

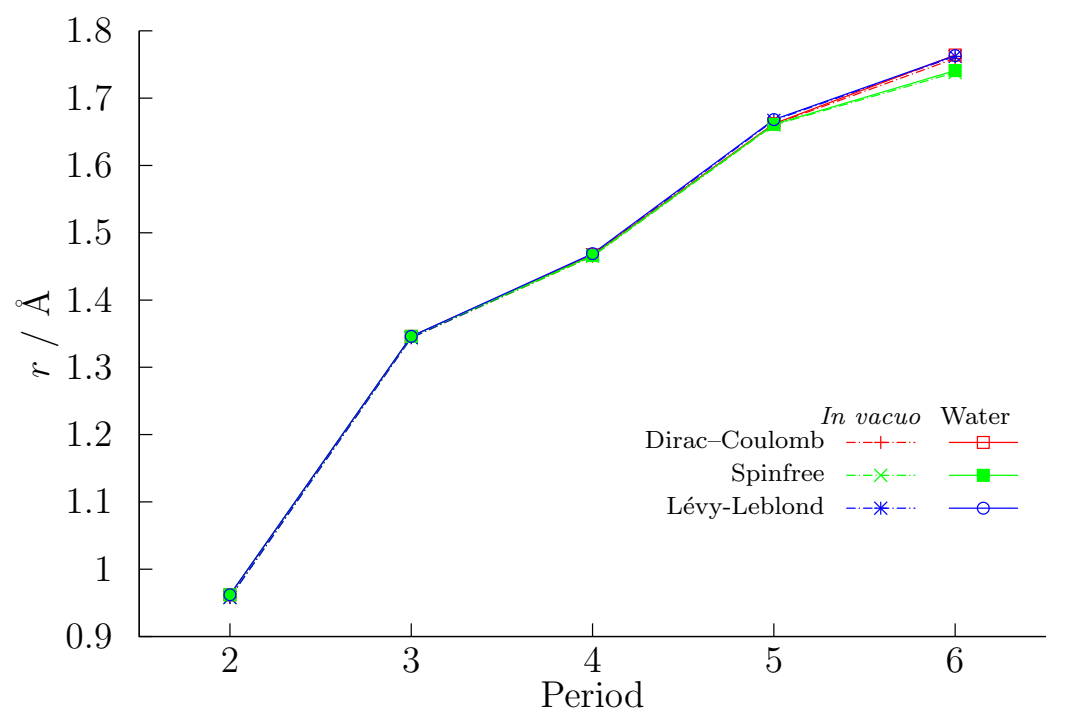

Figure 3: Optimized bond lengths at the DFT/PBE0 level of theory. Dot-dashed lines: in vacuo calculations. Solid lines: water calculations.

Figure 5 shows a uniform trend of decreasing dipole moment with the period, which clearly correlates with the reduction of the electronegativity of the central atom when going down the group 94 . From projection analysis ${ }^{100}$ we accordingly find at the DC/PBE0/vacuum 


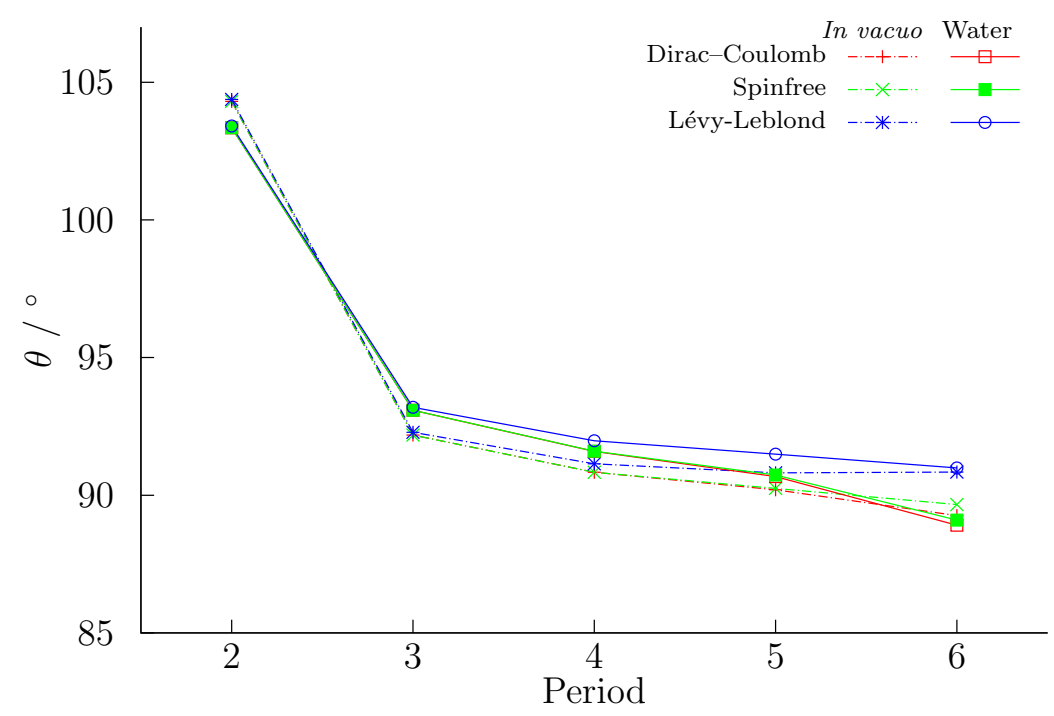

Figure 4: Optimized bond angles at the DFT/PBE0 level of theory. Dot-dashed lines: in vacuo calculations. Solid lines: water calculations.

level a charge of $-0.98 e$ on oxygen in $\mathrm{H}_{2} \mathrm{O}\left(\mu_{z}=+1.923 \mathrm{D}\right)$, whereas the corresponding charge on polonium in $\mathrm{H}_{2} \mathrm{Po}\left(\mu_{z}=-0.577 \mathrm{D}\right)$ is $+0.07 e$. The situation is somewhat more complex, though, because at the non-relativistic level the corresponding charge on polonium is almost the same $(+0.11 e)$, but now the dipole moment is positive $\left(\mu_{z}=+0.233 \mathrm{D}\right)$. This means that the inclusion of relativity switches the sign of the dipole moment in $\mathrm{H}_{2} \mathrm{Po}$, although the molecular geometry and atomic charges hardly change. In order to better understand this seeming paradox, we first recall that the electronic and nuclear contributions separately depend on the origin. Placing the origin of the dipole moment on the central atom, the nuclear contribution from polonium is identically zero, and the electronic contribution is essentially limited to four valence orbitals, associated with the two bonds and two lone pairs. After Pipek-Mezey localization we find that the weight of polonium in the bonding orbitals is $46.6 \%, 47.6 \%$ and $45.8 \%$ at the Dirac-Coulomb, spin-free and non-relativistic levels, respectively, showing that the polarity of the bonds is essentially independent of the choice of Hamiltonian, thus further adding to the enigma. However, the matrix elements of the $6 s$ and $6 p_{z}$ orbitals over the $\mu_{z}$-operator are significantly reduced with the introduction of scalar relativity, due to orbital contraction. This affects the lone pair orbitals more than 
the bonding ones: their contribution to the $z$-component of the dipole moment is thereby reduced from $4.78 \mathrm{D}$ to $3.40 \mathrm{D}$, whereas the contribution from the two bonding orbitals to the $z$-component of the dipole moment changes from $-16.26 \mathrm{D}$ to $-15.26 \mathrm{D}$. This explains the change of sign of the dipole moment. With the introduction of spin-orbit coupling the $6 p_{3 / 2}$ and $6 p_{1 / 2}$ components expand and contract, respectively, with respect to the spinfree $6 p$ orbital, but the sign of the dipole moment is conserved.

The inclusion of solvent significantly increases the magnitude of dipole moments for all species and with respect to all Hamiltonians included, a trend already present in Onsager's model ${ }^{115}$. Since the molecular geometries of the studied molecules are only slightly affected, this is clearly an electronic effect. For $\mathrm{H}_{2} \mathrm{Po}$ at the DC/PBE0 level the dipole moment changes from $-0.577 \mathrm{D}$ to $-0.842 \mathrm{D}$, indicating an electronic charge transfer to the hydrogens. From projection analysis we indeed find that the polonium charge increases slightly $(+0.07 e \rightarrow$ $+0.08 e)$, whereas the polarity of the bonds hardly changes.

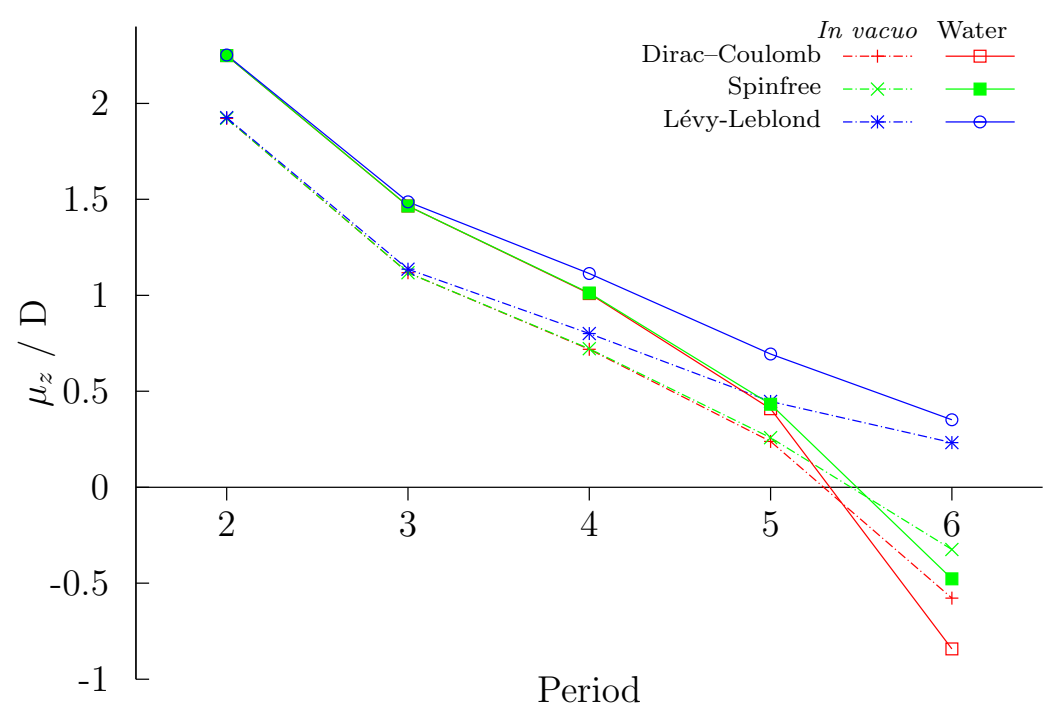

Figure 5: z-component of the electric dipole moment at the DFT/PBE0 level of theory. Dot-dashed lines: in vacuo calculations. Solid lines: water calculations.

Since bond lengths are affected neither by relativity nor by solvation, the solvent effect on these species is neatly summarized by saying that the existing charge separation is enhanced. This is strikingly demonstrated by the electrostatic potential maps in Figure 6] where the 
hydrogens of $\mathrm{H}_{2} \mathrm{O}\left(\mathrm{H}_{2} \mathrm{Po}\right)$ are seen to become more positive (negative), although it should be kept in mind that the electrostatic potential in a point is a weighted average of the charge density over all space. 116

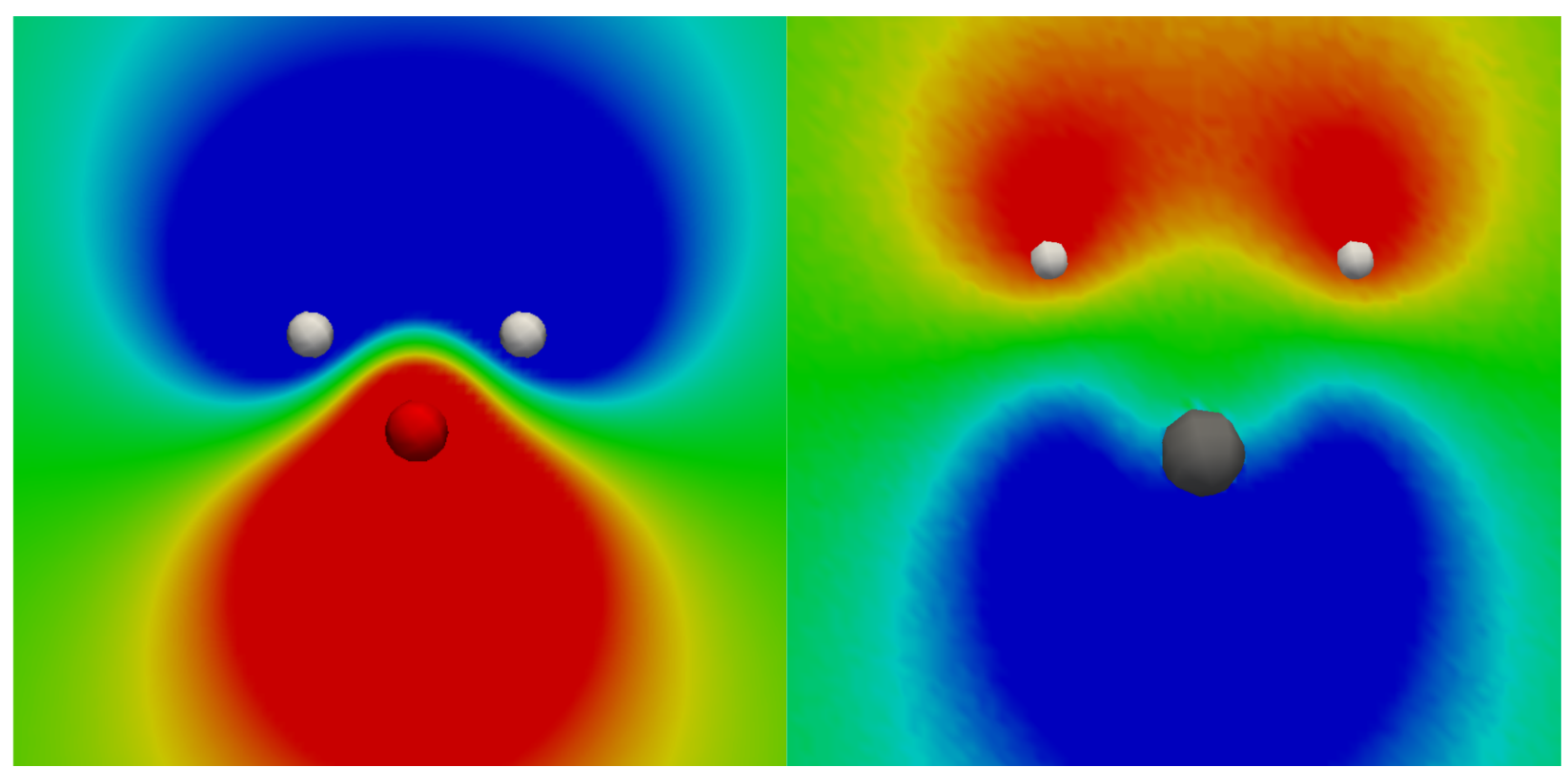

(a) $\mathrm{H}_{2} \mathrm{O}: \mathrm{MEP} \mathrm{PCM}_{-\mathrm{DC}}-\mathrm{MEP}_{\mathrm{DC}}$

(b) $\mathrm{H}_{2}$ Po: $\mathrm{MEP}_{\mathrm{PCM}-\mathrm{DC}}-\mathrm{MEP}_{\mathrm{DC}}$

Figure 6: Molecular electrostatic potential (MEP) maps for $\mathrm{H}_{2} \mathrm{O}$ and $\mathrm{H}_{2} \mathrm{Po}$ at the $\mathrm{DFT} / \mathrm{PBE} 0$ level of theory. The isocontours are in the range $[-0.02,0.02] E_{\mathrm{h}} e^{-1}$ and are color-coded from red to blue. The values plotted are differences between the values obtained for the Hamiltonians referred to in the subcaptions. The geometry optimized in vacuo, using the Dirac-Coulomb Hamiltonian as reference. PCM-DC: Dirac-Coulomb in water, DC: Dirac-Coulomb in vacuo.

\subsection{Electric dipole moment polarizabilities}

The components of the static electric dipole polarizability tensor are defined in terms of the linear response function as ${ }^{114}$, i.e. as the second derivative of the (free) energy with respect to an applied electric field $\boldsymbol{F}$ :

$$
\alpha_{I J}=-\left\langle\left\langle\mu_{I} ; \mu_{J}\right\rangle\right\rangle=-\left.\frac{\partial^{2} E(\boldsymbol{F})}{\partial F_{I} \partial F_{J}}\right|_{\boldsymbol{F}=0}
$$


where $\mu_{I}$ is the $I$-th component of the electric dipole operator. The isotropic part is

$$
\alpha_{\mathrm{iso}}=\frac{1}{3}\left(\alpha_{x x}+\alpha_{y y}+\alpha_{z z}\right)
$$

whereas the anisotropic part is defined as

$$
\alpha_{\text {aniso }}=\frac{1}{\sqrt{2}}\left[\left(\alpha_{x x}-\alpha_{y y}\right)^{2}+\left(\alpha_{x x}-\alpha_{z z}\right)^{2}+\left(\alpha_{y y}-\alpha_{z z}\right)^{2}+6\left(\alpha_{x y}^{2}+\alpha_{x z}^{2}+\alpha_{y z}^{2}\right)\right]^{1 / 2}
$$

In the present case the off-diagonal components of the polarizability tensor are zero by symmetry. Table 4 summarizes results obtained for the diagonal components as well as $\alpha_{\text {iso }}$ and $\alpha_{\text {aniso. }}$ In Figure $7 \alpha_{\text {iso }}$ is shown as a function of the period, at the DFT/PBE0 level of theory. A striking feature is the almost complete lack of relativistic effects on the electric dipole polarizability. This can, however, be understood from the connection between $\alpha_{\text {iso }}$ and molecular volume. In fact, from Figure 7 one may apprehend the more compact molecular structure of spin-free $\mathrm{H}_{2} \mathrm{Po}$ compared to the non-relativistic and fully relativistic counterparts. It should be noted, though, that this observation is far from general. Whenever relativity significantly modifies the spatial extent of valence orbitals, one may also expect large relativistic effects on the electric dipole polarizability, as reported for example for $\operatorname{HgS}^{117}$.

For the solvated systems, $\alpha_{\text {iso }}$ is always significantly greater $(\simeq 70 \%)$ than in vacuo. This is not unexpected as in solvent a greater charge separation was observed from the trends in dipole moments. The modification by the inclusion of solvent of individual components of the polarizability tensor is not uniform, though, and leads to less obvious trends for the polarizability anisotropy which are harder to rationalize, as seen from Figure 8. The significant change in the the polarizability anisotropy at the spinfree PBE0 level upon inclusion of solvent arises from the $90 \%$ increase of the $\alpha_{y y}$ (out-of-plane) component compared to a $60 \%$ increase of $\alpha_{x x}$ and $\alpha_{z z}$. 


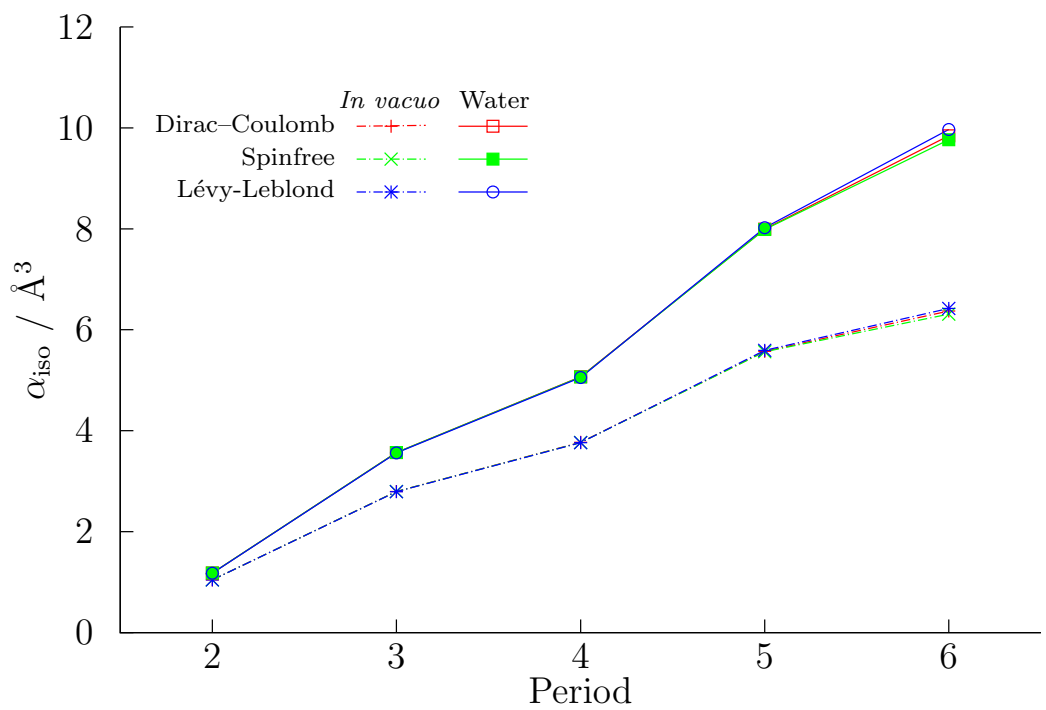

Figure 7: Isotropic electric dipole polarizability at the DFT/PBE0 of theory. Dot-dashed lines: in vacuo calculations. Solid lines: water calculations.

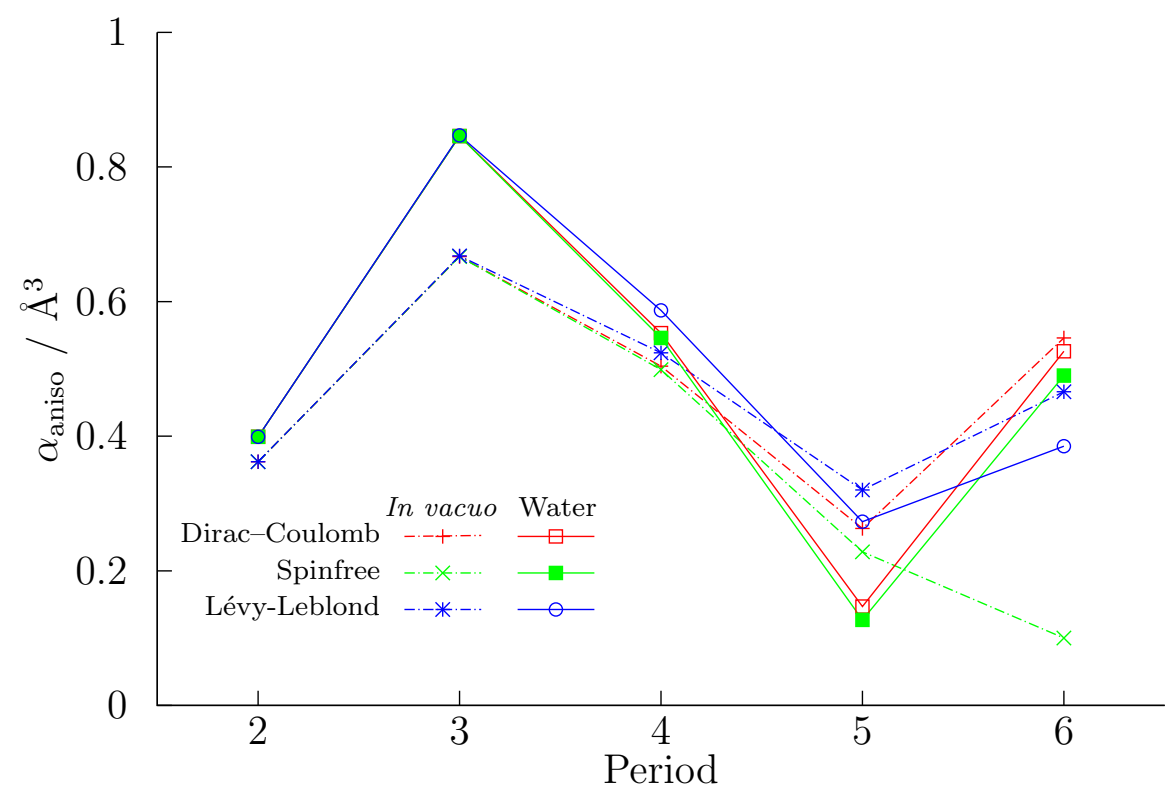

Figure 8: Anisotropic electric dipole polarizability at the DFT/PBE0 of theory. Dot-dashed lines: in vacuo calculations. Solid lines: water calculations. 
Table 4: Isotropic, anisotropic and Cartesian components of the electric dipole polarizability tensor. All values are reported in $\AA^{3}$.

\begin{tabular}{|c|c|c|c|c|c|c|c|c|c|c|c|c|c|c|c|c|c|}
\hline & & & \multicolumn{5}{|c|}{ Dirac-Coulomb } & \multicolumn{5}{|c|}{ Spin-free } & \multicolumn{5}{|c|}{ Lévy-Leblond } \\
\hline & & & $\alpha_{\text {iso }}$ & $\alpha_{\text {aniso }}$ & $\alpha_{x x}$ & $\alpha_{y y}$ & $\alpha_{z z}$ & $\alpha_{\text {iso }}$ & $\alpha_{\text {aniso }}$ & $\alpha_{x x}$ & $\alpha_{y y}$ & $\alpha_{z z}$ & $\alpha_{\text {iso }}$ & $\alpha_{\text {aniso }}$ & $\alpha_{x x}$ & $\alpha_{y y}$ & $\alpha_{z z}$ \\
\hline \multirow{10}{*}{ Hartree-Fock } & \multirow{5}{*}{ In vacuo } & $\mathrm{H}_{2} \mathrm{O}$ & 0.959 & 0.334 & 1.147 & 0.762 & 0.967 & 0.959 & 0.334 & 1.147 & 0.762 & 0.967 & 0.958 & 0.333 & 1.146 & 0.762 & 0.965 \\
\hline & & $\mathrm{H}_{2} \mathrm{~S}$ & 2.715 & 0.654 & 3.021 & 2.293 & 2.831 & 2.715 & 0.654 & 3.021 & 2.293 & 2.831 & 2.712 & 0.655 & 3.020 & 2.289 & 2.825 \\
\hline & & $\mathrm{H}_{2} \mathrm{Se}$ & 3.657 & 0.558 & 3.927 & 3.300 & 3.743 & 3.655 & 0.554 & 3.924 & 3.301 & 3.741 & 3.654 & 0.572 & 3.938 & 3.292 & 3.733 \\
\hline & & $\mathrm{H}_{2} \mathrm{Te}$ & 5.427 & 0.393 & 5.649 & 5.196 & 5.436 & 5.421 & 0.364 & 5.632 & 5.211 & 5.420 & 5.464 & 0.439 & 5.731 & 5.227 & 5.434 \\
\hline & & $\mathrm{H}_{2}^{2} \mathrm{Po}$ & 6.201 & 0.729 & 6.490 & 5.718 & 6.394 & 6.130 & 0.312 & 6.288 & 5.934 & 6.167 & 6.289 & 0.607 & 6.643 & 5.942 & 6.282 \\
\hline & \multirow{5}{*}{ Water } & $\mathrm{H}_{2} \mathrm{O}$ & 1.075 & 0.363 & 1.277 & 0.858 & 1.091 & 1.075 & 0.363 & 1.277 & 0.858 & 1.091 & 1.074 & 0.363 & 1.275 & 0.857 & $\overline{1.090}$ \\
\hline & & $\mathrm{H}_{2} \mathrm{~S}$ & 3.443 & 0.832 & 3.850 & 2.913 & 3.567 & 3.443 & 0.832 & 3.850 & 2.913 & 3.567 & 3.437 & 0.833 & 3.847 & 2.907 & 3.557 \\
\hline & & $\mathrm{H}_{2} \mathrm{Se}$ & 4.871 & 0.667 & 5.225 & 4.461 & 4.925 & 4.868 & 0.661 & 5.220 & 4.463 & 4.922 & 4.864 & 0.691 & 5.240 & 4.445 & 4.906 \\
\hline & & $\mathrm{H}_{2} \mathrm{Te}$ & 7.696 & 0.449 & 7.992 & 7.508 & 7.587 & 7.688 & 0.412 & 7.962 & 7.537 & 7.565 & 7.755 & 0.559 & 8.128 & 7.562 & 7.575 \\
\hline & & $\mathrm{H}_{2} \mathrm{Po}$ & 9.452 & 1.010 & 9.858 & 8.784 & 9.715 & 9.346 & 0.169 & 9.455 & 9.312 & 9.270 & 9.631 & 0.790 & 10.152 & 9.305 & 9.436 \\
\hline \multirow{10}{*}{ DFT/PBE0 } & \multirow{5}{*}{ In vacuo } & $\mathrm{H}_{2} \mathrm{O}$ & 1.046 & 0.362 & 1.241 & 0.826 & 1.071 & 1.046 & 0.362 & 1.241 & 0.826 & 1.071 & 1.045 & 0.362 & 1.240 & 0.825 & 1.069 \\
\hline & & $\mathrm{H}_{2} \mathrm{~S}$ & 2.796 & 0.667 & 3.072 & 2.356 & 2.961 & 2.796 & 0.667 & 3.071 & 2.356 & 2.961 & 2.792 & 0.668 & 3.069 & 2.351 & 2.955 \\
\hline & & $\mathrm{H}_{2} \mathrm{Se}$ & 3.770 & 0.504 & 3.975 & 3.437 & 3.898 & 3.769 & 0.499 & 3.972 & 3.439 & 3.895 & 3.764 & 0.524 & 3.984 & 3.419 & 3.890 \\
\hline & & $\mathrm{H}_{2} \mathrm{Te}$ & 5.573 & 0.263 & 5.693 & 5.403 & 5.624 & 5.567 & 0.228 & 5.674 & 5.420 & 5.607 & 5.593 & 0.320 & 5.760 & 5.395 & 5.625 \\
\hline & & $\mathrm{H}_{2} \mathrm{Po}$ & 6.372 & 0.546 & 6.550 & 6.008 & 6.557 & 6.310 & 0.100 & 6.350 & 6.243 & 6.336 & 6.423 & 0.466 & 6.656 & 6.128 & 6.485 \\
\hline & \multirow{5}{*}{ Water } & $\mathrm{H}_{2} \mathrm{O}$ & 1.180 & 0.399 & 1.390 & 0.933 & 1.216 & 1.180 & 0.399 & 1.390 & 0.933 & 1.216 & 1.178 & 0.399 & 1.388 & 0.932 & 1.214 \\
\hline & & $\mathrm{H}_{2} \mathrm{~S}$ & 3.567 & 0.846 & 3.930 & 3.012 & 3.760 & 3.567 & 0.846 & 3.930 & 3.012 & 3.760 & 3.560 & 0.847 & 3.926 & 3.005 & 3.750 \\
\hline & & $\mathrm{H}_{2} \mathrm{Se}$ & 5.069 & 0.553 & 5.317 & 4.709 & 5.182 & 5.067 & 0.546 & 5.312 & 4.712 & 5.178 & 5.056 & 0.587 & 5.327 & 4.676 & 5.164 \\
\hline & & $\mathrm{H}_{2} \mathrm{Te}$ & 7.995 & 0.147 & 8.092 & 7.954 & 7.939 & 7.986 & 0.127 & 8.059 & 7.989 & 7.912 & 8.023 & 0.273 & 8.204 & 7.930 & 7.933 \\
\hline & & $\mathrm{H}_{2} \mathrm{Po}$ & 9.843 & 0.526 & 10.014 & 9.492 & 10.023 & 9.766 & 0.490 & 9.592 & 10.092 & 9.614 & 9.969 & 0.385 & 10.220 & 9.797 & 9.891 \\
\hline
\end{tabular}




\subsection{Choice of solvent}

In the above calculations water, with relative dielectric constant $\varepsilon_{r}=78.39$, was selected as solvent. It may be objected that specific interactions of a protic solvent such as water and the solute will be important and are not captured by a continuum model such as PCM. However, this will depend to what extent specific interactions such as hydrogen bonding are important for the property under study. Continuum models such as PCM have been widely applied to describe aqueous solution, in most cases with quite satisfactory results $\underline{26150}$. To rigorously demonstrate shortcomings of our relativistic results, due to the persistence and importance of specific interactions over time, would require molecular dynamics simulations

with a statistically significant sampling 21118 , a computational protocol which is not presently available at the 4-component relativistic level. It should also to be noted that the present implementation only includes contributions of electrostatic origin, neglecting dispersion, repulsion $\frac{119}{19}$ and cavitation $\frac{120}{120}$ contributions: a fair comparison between continuum and explicit models has to take into account all energy contributions.

In figure 9 we display the electric dipole moment and isotropic dipole polarizability of $\mathrm{H}_{2} \mathrm{Po}$, relative to vacuum values, as a function of relative permittivity $\varepsilon_{r}$. The vacuum optimized molecular geometry was employed, since solvent effects on geometries were found to be small. Given the solutes considered in this study we do not expect nonlinearities 121 in the solvent effect, hence lower polarity solvents should yield a reduced effect with values that are "bracketed" by the gas-phase and water ones. This is indeed what we observe, as well as the well-known saturation of dielectric response $\mathrm{e}^{122 \mid 123}$. The relative difference of the observables can be perfectly fitted (correlation coefficient 1) to a linear rational function, that is

$$
X\left(\varepsilon_{r}\right)-X(1)=\frac{\varepsilon_{r}-1}{a \varepsilon_{r}+b} X(1) ; \quad X=\mu_{z}, \alpha_{\text {iso }}
$$

For $\mathrm{H}_{2} \mathrm{Po}$ the values $(a, b)$ of the fit coefficients are $(2.339,3.140)$ and $(1.797,2.345)$ for $\mu_{z}$ and $\alpha_{\text {iso }}$, respectively. These values are system-dependent; with water as solute we obtain 


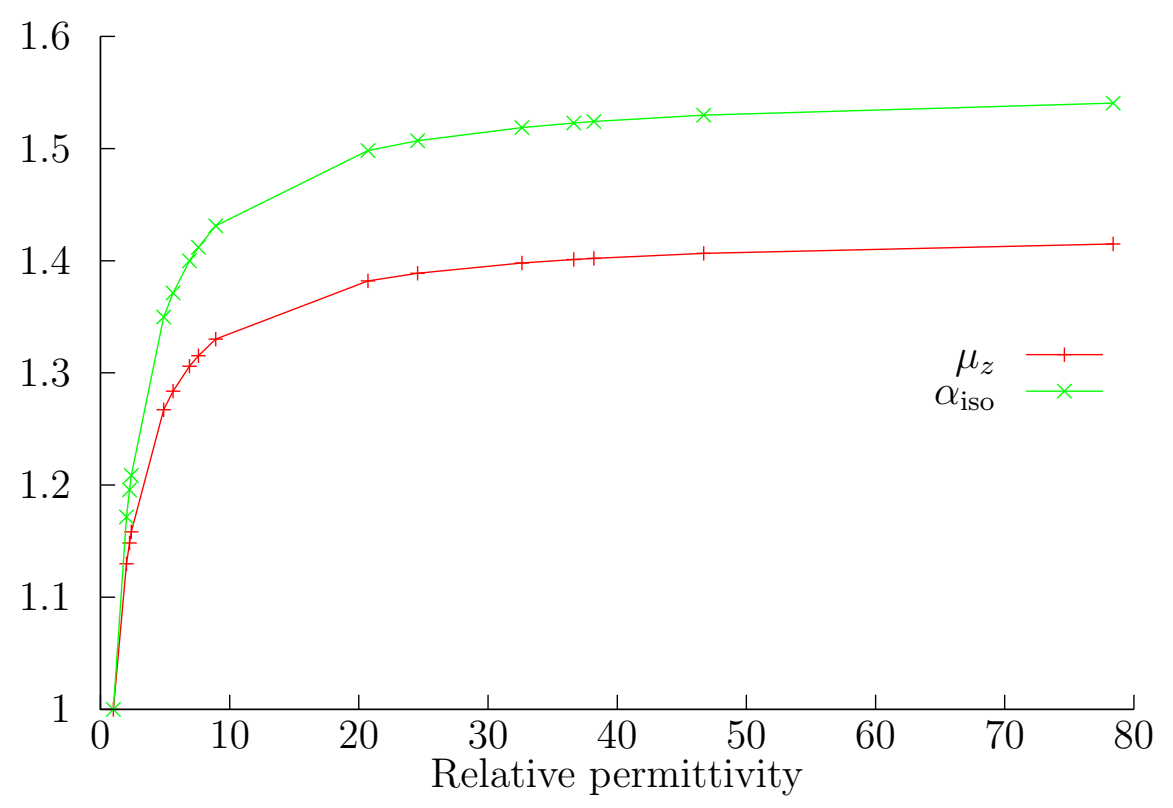

Figure 9: Electric dipole and isotropic dipole polarizability of $\mathrm{H}_{2} \mathrm{Po}$, relative to vacuum values, as a function of relative permittivity $\varepsilon_{r}$.

$(6.150,3.942)$ and $(8.084,5.251)$.

\section{Conclusions}

A detailed derivation of the Polarizable Continuum Model for 4-component Hartree-Fock and Kohn-Sham calculations has been presented. The derivation of the first-order response equation including the contributions from a polarizable continuum has also been detailed. The algorithm implemented has been described and in particular the advantages of the modular programming paradigm adopted have been elucidated. This new functionality implemented in the DIRAC program package will be made available in the DIRAC14 release. We would like to stress the importance of modularity for the work here presented. Use of the flexible API library PCMSolver has effectively enabled us to use a tested and standardized implementation of the PCM related tasks in the more general framework of 4-component electronic-structure theory. PCMSolver clearly implements the emerging ideas in modern programming techniques, such as abstraction, data hiding and, above all, code reusability. 
The few results summarized in this paper show some of the many potential applications of the 4-component PCM-SCF scheme. Calculation of excitation energies using our LRPCM-SCF algorithm is possible with a small additional coding effort, in order to properly take into account the effect of nonequilibrium solvation on the excitation process $\frac{11}{\text {. Fur- }}$ ther developments of the work here presented regard the calculation of parameters relevant for magnetic spectroscopies and the extension to 2-component Hamiltonian models $\frac{63}{}$. Both lines of development are currently being investigated.

\section{Acknowledgement}

This paper is dedicated to Prof. Jacopo Tomasi on the occasion of his 80th birthday. One of the authors (L.F.) has had the privilege to graduate under Tomasi's supervision, appreciating his passion for science, his curiosity for new developments and his immense knowledge of the scientific literature. The authors would like to thank Prof. Benedetta Mennucci for useful discussions. R.D.R. gratefully acknowledges the financial support from the Erasmus Lifelong Learning Programme during his stay in Toulouse. This work has been supported by the Research Council of Norway through a Centre of Excellence Grant (Grant No. 179568/V30) and through a NOTUR allocation of computer resources (Grant No. NN4654K).

\section{References}

(1) Dirac, P. A. M. Quantum Mechanics of Many-Electron Systems. Proc. Roy. Soc. Lond. A 1929, 123, 714-733.

(2) Kutzelnigg, W. Perspective on "Quantum Mechanics of Many-Electron Systems". Theor. Chem. Acc. 2000, 103, 182-186.

(3) Markus, R.; Wolf, A. Relativistic Quantum Chemistry; Wiley-VCH Verlag GmbH \& Co. KGaA, 2009. 
(4) Pyykkö, P. Relativistic Effects in Chemistry: More Common Than You Thought. Annu. Rev. Phys. Chem. 2012, 63, 45-64.

(5) Glantschnig, K.; Ambrosch-Draxl, C. Relativistic Effects on the Linear Optical Properties of Au, Pt, Pb and W. New. J. Phys. 2010, 12, 103048-103064.

(6) Reichardt, C.; Welton, T. Solvents and Solvent Effects in Organic Chemistry; WileyVCH Verlag GmbH \& Co. KGaA, 2010.

(7) Hansen, J.-P.; McDonald, I. R. Theory of Simple Liquids; Academic Press: Burlington, 2006.

(8) Hill, T. L. An Introduction to Statistical Thermodynamics; Dover Publications, Inc.: New York, 1986.

(9) Tomasi, J. In Continuum Solvation Models in Chemical Physics; Mennucci, B., Cammi, R., Eds.; John Wiley \& Sons, Ltd, 2007; pp 1-28.

(10) Mennucci, B., Cammi, R., Eds. Continuum Solvation Models in Chemical Physics; John Wiley \& Sons, Ltd, 2007.

(11) Tomasi, J.; Mennucci, B.; Cammi, R. Quantum Mechanical Continuum Solvation Models. Chem. Rev. 2005, 105, 2999-3094.

(12) Senn, H. M.; Thiel, W. QM/MM Methods for Biomolecular Systems. Angew. Chem. Int. Edit. 2009, 48, 1198-1229.

(13) Curutchet, C.; Muñoz Losa, A.; Monti, S.; Kongsted, J.; Scholes, G. D.; Mennucci, B. Electronic Energy Transfer in Condensed Phase Studied by a Polarizable QM/MM Model. J. Chem. Theory Comput. 2009, 5, 1838-1848.

(14) Olsen, J. M.; Aidas, K.; Kongsted, J. Excited States in Solution through Polarizable Embedding. J. Chem. Theory Comput. 2010, 6, 3721-3734. 
(15) Steindal, A. H.; Ruud, K.; Frediani, L.; Aidas, K.; Kongsted, J. Excitation Energies in Solution: The Fully Polarizable QM/MM/PCM Method. J. Phys. Chem. B 2011, $115,3027-3037$.

(16) Lipparini, F.; Barone, V. Polarizable Force Fields and Polarizable Continuum Model: A Fluctuating Charges/PCM Approach. 1. Theory and Implementation. J. Chem. Theory Comput. 2011, 7, 3711-3724.

(17) Caprasecca, S.; Curutchet, C.; Mennucci, B. Toward a Unified Modeling of Environment and Bridge-Mediated Contributions to Electronic Energy Transfer: A Fully Polarizable QM/MM/PCM Approach. J. Chem. Theory Comput. 2012, 8, 4462-4473.

(18) Morss, L., Edelstein, N., Fuger, J., Katz, J., Eds. The Chemistry of the Actinide and Transactinide Elements; Springer, 2011.

(19) Vallet, V.; Macak, P.; Wahlgren, U.; Grenthe, I. Actinide Chemistry in Solution, Quantum Chemical Methods and Models. Theor. Chem. Acc. 2006, 115, 145-160.

(20) Schreckenbach, G.; Shamov, G. A. Theoretical Actinide Molecular Science. Acc. Chem. Res. 2010, 43, 19-29.

(21) Mennucci, B.; Martínez, J.; Tomasi, J. Solvent Effects on Nuclear Shieldings: Continuum or Discrete Solvation Models to Treat Hydrogen Bond and Polarity Effects? J. Phys. Chem. A 2001, 105, 7287-7296.

(22) Vicha, J.; Patzschke, M.; Marek, R. A Relativistic DFT Methodology for Calculating the Structures and NMR Chemical Shifts of Octahedral Platinum and Iridium Complexes. Phys. Chem. Chem. Phys. 2013, 15, 7740-7754.

(23) Standara, S.; Malinakova, K.; Marek, R.; Marek, J.; Hocek, M.; Vaara, J.; Straka, M. Understanding the NMR Chemical Shifts for 6-Halopurines: Role of Structure, Solvent and Relativistic Effects. Phys. Chem. Chem. Phys. 2010, 12, 5126-5139. 
(24) Moncho, S.; Autschbach, J. Relativistic Zeroth-Order Regular Approximation Combined with Nonhybrid and Hybrid Density Functional Theory: Performance for NMR Indirect Nuclear Spin-Spin Coupling in Heavy Metal Compounds. J. Chem. Theory Comput. 2010, 6, 223-234.

(25) Hay, P. J.; Martin, R. L.; Schreckenbach, G. Theoretical Studies of the Properties and Solution Chemistry of $\mathrm{AnO}_{2}^{2+}$ and $\mathrm{AnO}_{2}^{+}$Aquo Complexes for $\mathrm{An}=\mathrm{U}, \mathrm{Np}$, and $\mathrm{Pu}$. J. Phys. Chem. A 2000, 104, 6259-6270.

(26) Vallet, V.; Wahlgren, U.; Schimmelpfennig, B.; Moll, H.; Szabó, Z.; Grenthe, I. Solvent Effects on Uranium(VI) Fluoride and Hydroxide Complexes Studied by EXAFS and Quantum Chemistry. Inorg. Chem. 2001, 40, 3516-3525.

(27) Siboulet, B.; Marsden, C. J.; Vitorge, P. A Theoretical Study of Uranyl Solvation: Explicit Modelling of the Second Hydration Sphere by Quantum Mechanical Methods. Chem. Phys. 2006, 326, 289-296.

(28) Barakat, K. A.; Cundari, T. R.; Rabaâ, H.; Omary, M. A. Disproportionation of Gold(II) Complexes: A Density Functional Study of Ligand and Solvent Effects. J. Phys. Chem. B 2006, 110, 14645-14651.

(29) Liao, Y.; Ma, J. Stacking and Solvent Effects on the Electronic and Optical Properties of Gold and Mercury Acetylide Aggregations: A Theoretical Study. Organometallics 2008, 27, 4636-4648.

(30) Periyasamy, G.; Remacle, F. Ligand and Solvation Effects on the Electronic Properties of $\mathrm{Au}_{55}$ Clusters: A Density Functional Theory Study. Nano Lett. 2009, 9, 3007-3011.

(31) Vallet, V.; Grenthe, I. On the Structure and Relative Stability of Uranyl(VI) Sulfate Complexes in Solution. Comptes Rendus Chim. 2007, 10, 905-915. 
(32) Wå hlin, P.; Schimmelpfennig, B.; Wahlgren, U.; Grenthe, I.; Vallet, V. On the Combined use of Discrete Solvent Models and Continuum Descriptions of Solvent Effects in Ligand Exchange Reactions: a Case Study of the Uranyl(VI) Aquo Ion. Theor. Chem. Acc. 2009, 124, 377-384.

(33) van Lenthe, E.; Baerends, E. J.; Snijders, J. G. Relativistic Total Energy Using Regular Approximations. J. Chem. Phys. 1994, 101, 9783-9792.

(34) Fuchs, M. S. K.; Shor, A. M.; Rösch, N. The Hydration of the Uranyl Dication: Incorporation of Solvent Effects in Parallel Density Functional Calculations with the Program PARAGAUSS. Int. J. Quantum Chem. 2002, 86, 487-501.

(35) Moskaleva, L. V.; Krüger, S.; Spörl, A.; Rösch, N. Role of Solvation in the Reduction of the Uranyl Dication by Water: A Density Functional Study. Inorg. Chem. 2004, 43, 4080-4090.

(36) Shamov, G. A.; Schreckenbach, G. Density Functional Studies of Actinyl Aquo Complexes Studied Using Small-Core Effective Core Potentials and a Scalar FourComponent Relativistic Method. J. Phys. Chem. A 2005, 109, 10961-10974.

(37) Páez-Hernández, D.; Ramírez-Tagle, R.; Codorniu-Hernández, E.; MonteroCabrera, L. A.; Arratia-Pérez, R. Quantum Relativistic Investigation about the Coordination and Bonding Effects of Different Ligands on Uranyl Complexes. Polyhedron 2010, 29, 975-984.

(38) Odoh, S. O.; Walker, S. M.; Meier, M.; Stetefeld, J.; Schreckenbach, G. QM and QM/MM Studies of Uranyl Fluorides in the Gas and Aqueous Phases and in the Hydrophobic Cavities of Tetrabrachion. Inorg. Chem. 2011, 50, 3141-3152.

(39) Pan, Q.-J.; Odoh, S. O.; Schreckenbach, G.; Arnold, P. L.; Love, J. B. Theoretical Exploration of Uranyl Complexes of a Designed Polypyrrolic Macrocycle: Struc- 
ture/Property Effects of Hinge Size on Pacman-Shaped Complexes. Dalton Trans. 2012, 41, 8878-8885.

(40) Schlosser, F.; Moskaleva, L. V.; Kremleva, A.; Krüger, S.; Rösch, N. Comparative Density Functional Study of the Complexes $\left[\mathrm{UO}_{2}\left(\mathrm{CO}_{3}\right)_{3}\right]^{4-}$ and $\left[\left(\mathrm{UO}_{2}\right)_{3}\left(\mathrm{CO}_{3}\right)_{6}\right]^{6-}$ in Aqueous Solution. Dalton Trans. 2010, 39, 5705-5712.

(41) Klamt, A.; Schüürmann, G. COSMO: A New Approach to Dielectric Screening in Solvents with Explicit Expressions for the Screening Energy and its Gradient. J. Chem. Soc., Perkin Trans. 2 1993, 799-805.

(42) Pye, C. C.; Ziegler, T. An Implementation of the Conductor-Like Screening Model of Solvation within the Amsterdam Density Functional Package. Theor. Chem. Acc. 1999, 101, 396-408.

(43) Cossi, M.; Rega, N.; Scalmani, G.; Barone, V. Energies, Structures, and Electronic Properties of Molecules in Solution with the C-PCM Solvation Model. J. Comput. Chem. 2003, 24, 669-81.

(44) Infante, I.; Visscher, L. QM/MM Study of Aqueous Solvation of the Uranyl Fluoride $\left[\mathrm{UO}_{2} \mathrm{~F}_{4}^{2-}\right]$ Complex. J. Comput. Chem. 2004, 25, 386-392.

(45) Infante, I.; van Stralen, B.; Visscher, L. A QM/MM Study on the Aqueous Solvation of the Tetrahydroxouranylate $\left[\mathrm{UO}_{2}(\mathrm{OH})_{4}\right]^{2-}$ Complex Ion. J. Comput. Chem. 2006, 27, 1156-1162.

(46) Sinnecker, S.; Neese, F. QM/MM Calculations with DFT for Taking into Account Protein Effects on the EPR and Optical Spectra of Metalloproteins. Plastocyanin as a Case Study. J. Comput. Chem. 2006, 27, 1463-1475.

(47) Chaumont, A.; Wipff, G. Solvation of Uranyl(II), Europium(III) and Europium(II) 
Cations in Basic Room-Temperature Ionic Liquids: A Theoretical Study. Chem. Eur. J. 2004, 10, 3919-3930.

(48) Autschbach, J.; Ziegler, T. Solvent Effects on Heavy Atom Nuclear Spin-Spin Coupling Constants: A Theoretical Study of Hg-C and Pt-P Couplings. J. Am. Chem. Soc. 2001, 123, 3341-9.

(49) Autschbach, J.; Ziegler, T. A Theoretical Investigation of the Remarkable Nuclear Spin-Spin Coupling Pattern in $\left[(\mathrm{NC})_{5} \mathrm{Pt}-\mathrm{Tl}(\mathrm{CN})\right]^{-}$. J. Am. Chem. Soc. 2001, 123, $5320-4$.

(50) Autschbach, J.; Le Guennic, B. A Theoretical Study of the NMR Spin-Spin Coupling Constants of the Complexes $\left[(\mathrm{NC})_{5} \mathrm{Pt}-\mathrm{Tl}(\mathrm{CN})_{n}\right]^{n-}(n=0-3)$ and $\left[(\mathrm{NC})_{5} \mathrm{Pt}-\right.$ Tl-Pt $\left.(\mathrm{CN})_{5}\right]^{3-}$ : A Lesson on Environmental Effects. J. Am. Chem. Soc. 2003, 125, 13585-93.

(51) Autschbach, J.; Le Guennic, B. Solvent Effects on ${ }^{195} \mathrm{Pt}$ and ${ }^{205} \mathrm{Tl}$ NMR Chemical Shifts of the Complexes $\left[(\mathrm{NC})_{5} \mathrm{Pt}-\mathrm{Tl}(\mathrm{CN})_{n}\right]^{n-}(n=0-3)$, and $\left[(\mathrm{NC})_{5} \mathrm{Pt}-\mathrm{Tl}-\mathrm{Pt}(\mathrm{CN})_{5}\right]^{3-}$ Studied by Relativistic Density Functional Theory. Chem. Eur. J. 2004, 10, 25812589.

(52) Le Guennic, B.; Matsumoto, K.; Autschbach, J. NMR Properties of PlatinumThallium Bonded Complexes: Analysis of Relativistic Density Functional Theory Results. Magn. Reson. Chem. 2004, 42 Spec no, S99-S116.

(53) Chen, W.; Liu, F.; Matsumoto, K.; Autschbach, J.; Le Guennic, B.; Ziegler, T.; Maliarik, M.; Glaser, J. Spectral and Structural Characterization of Amidate-Bridged Platinum-Thallium Complexes with Strong Metal-Metal Bonds. Inorg. Chem. 2006, $45,4526-36$.

(54) Sterzel, M.; Autschbach, J. Toward an Accurate Determination of ${ }^{195} \mathrm{Pt}$ Chemical Shifts by Density Functional Computations: The Importance of Unspecific Solvent 
Effects and the Dependence of Pt Magnetic Shielding Constants on Structural Parameters. Inorg. Chem. 2006, 45, 3316-3324.

(55) Autschbach, J.; Sterzel, M. Molecular Dynamics Computational Study of the ${ }^{199} \mathrm{Hg}-$ ${ }^{199} \mathrm{Hg}$ NMR Spin-Spin Coupling Constants of $[\mathrm{Hg}-\mathrm{Hg}-\mathrm{Hg}]^{2+}$ in $\mathrm{SO}_{2}$ Solution. J. Am. Chem. Soc. 2007, 129, 11093-11099.

(56) Zheng, S.; Autschbach, J. Modeling of Heavy-Atom-Ligand NMR Spin-Spin Coupling in Solution: Molecular Dynamics Study and Natural Bond Orbital Analysis of Hg-C Coupling Constants. Chem. Eur. J. 2011, 17, 161-173.

(57) Bühl, M.; Wipff, G. Insights into Uranyl Chemistry from Molecular Dynamics Simulations. ChemPhysChem 2011, 12, 3095-105.

(58) Camellone, M. F.; Marx, D. Solvation of $\mathrm{Au}^{+}$versus $\mathrm{Au}^{0}$ in Aqueous Solution: Electronic Structure Governs Solvation Shell Patterns. Phys. Chem. Chem. Phys. 2012, $14,937-944$.

(59) Miertuš, S.; Scrocco, E.; Tomasi, J. Electrostatic Interaction of a Solute with a Continuum. A Direct Utilization of Ab Initio Molecular Potentials for the Prevision of Solvent Effects. Chem. Phys. 1981, 55, 117-129.

(60) DIRAC, a relativistic ab initio electronic structure program, Release DIRAC13 (2013), written by L. Visscher, H. J. Aa. Jensen, R. Bast, and T. Saue, (see http://www.diracprogram.org).

(61) PCMSolver, an Application Programming Interface for the Polarizable Continuum Model electrostatic problem, written by R. Di Remigio, L. Frediani and K. Mozgawa, (see http://pcmsolver.github.io/pcmsolver-doc).

(62) Whiffen, D. H. Expression of Results in Quantum Chemistry. Pure Appl. Chem. 1978, $50,75-79$. 
(63) Saue, T. Relativistic Hamiltonians for Chemistry: A Primer. ChemPhysChem 2011, 12, 3077-3094.

(64) Dyall, K. G.; Fægri, K., Jr. An Introduction to Relativistic Quantum Chemistry; Oxford University Press, 2007.

(65) Foldy, L. L.; Wouthuysen, S. A. On the Dirac Theory of Spin 1/2 Particles and its Non-Relativistic Limit. Phys. Rev. 1950, 78, 29-36.

(66) Itoh, T. Derivation of Nonrelativistic Hamiltonian for Electrons from Quantum Electrodynamics. Rev. Mod. Phys. 1965, 37, 159-165.

(67) Trond Saue, Principles and Applications of Relativistic Molecular Calculations. Ph.D. thesis, University of Oslo, 1996.

(68) Brown, G. E.; Ravenhall, D. G. On the Interaction of Two Electrons. Proc. Roy. Soc. Lond. A 1951, 208, 552-559.

(69) Sucher, J. Foundations of the Relativistic Theory of Many-Electron Atoms. Phys. Rev. A 1980, 22, 348-362.

(70) Dyall, K. G. An Exact Separation of the Spin-Free and Spin-Dependent Terms of the Dirac-Coulomb-Breit Hamiltonian. J. Chem. Phys. 1994, 100, 2118-2127.

(71) Lévy Leblond, J.-M. Nonrelativistic Particles and Wave Equations. Comm. Math. Phys. 1967, 6, 286-311.

(72) Cancès, E.; Mennucci, B. New Applications of Integral Equations Methods for Solvation Continuum Models: Ionic Solutions and Liquid Crystals. J. Math. Chem. 1998, 23, 309-326.

(73) Hsiao, G. C.; Wendland, W. L. In Boundary Integral Equations; Antman, S. S., Holmes, P., Sirovich, L., Sreenivasan, K., Eds.; Applied Mathematical Sciences; Springer Berlin Heidelberg, 2008; Vol. 164. 
(74) Wolfgang Hackbusch, Integral Equations - Theory and Numerical Treatment; Birkhaüser, 1995.

(75) Pomelli, C. S. In Continuum Solvation Models in Chemical Physics; Mennucci, B., Cammi, R., Eds.; John Wiley \& Sons, Ltd, 2007; pp 49-63.

(76) Sanhueza, J. E.; Tapia, O.; Laidlaw, W. G.; Trsic, M. On the Application of the Variational Principle to a Type of Nonlinear "Schrödinger Equation". J. Chem. Phys. 1979, 70, 3096-3098.

(77) Saue, T.; Helgaker, T. Four-Component Relativistic Kohn-Sham Theory. J. Comput. Chem. 2002, 23, 814-823.

(78) Eschrig, H. The Fundamentals of Density Functional Theory; Teubner, Stuttgart, 1996.

(79) Rajagopal, A. K.; Callaway, J. Inhomogeneous Electron Gas. Phys. Rev. B 1973, 7, 1912-1919.

(80) Helgaker, T.; Jørgensen, P.; Olsen, J. Molecular Electronic-Structure Theory; John Wiley \& Sons., 2000.

(81) Visscher, L. Approximate Molecular Relativistic Dirac-Coulomb Calculations Using a Simple Coulombic Correction. Theor. Chem. Acc. 1997, 98, 68-70.

(82) Salek, P.; Helgaker, T.; Saue, T. Linear Response at the 4-Component Relativistic Density-Functional Level: Application to the Frequency-Dependent Dipole Polarizability of $\mathrm{Hg}, \mathrm{AuH}$ and $\mathrm{PtH}_{2}$. Chem. Phys. 2005, 311, 187-201.

(83) Cammi, R.; Tomasi, J. Nonequilibrium Solvation Theory for the Polarizable Continuum Model: A New Formulation at the SCF Level with Application to the Case of the Frequency-Dependent Linear Electric Response Function. Int. J. Quantum Chem. $1995,56,465-474$. 
(84) Helgaker, T.; Coriani, S.; Jø rgensen, P.; Kristensen, K.; Olsen, J.; Ruud, K.; Hamiltonian, B. A. P.; Electronic, M.; Pauli, B. A. Recent Advances in Wave Function-Based Methods of Molecular-Property Calculations. Chem. Rev. 2012, 112, 543-631.

(85) Kauczor, J.; Jørgensen, P.; Norman, P. On the Efficiency of Algorithms for Solving Hartree-Fock and Kohn-Sham Response Equations. J. Chem. Theory Comput. 2011, 7, 1610-1630.

(86) Saue, T. In Relativistic Electronic Structure Theory; Schwerdtfeger, P., Ed.; Theoretical and Computational Chemistry; Elsevier, 2002; Vol. 11; pp 332-400.

(87) Saue, T.; Jensen, H. J. Aa. Linear Response at the 4-Component Relativistic Level: Application to the Frequency-Dependent Dipole Polarizabilities of the Coinage Metal Dimers. J. Chem. Phys. 2003, 118, 522-536.

(88) Dijkstra, E. W. The Structure of the "THE"-multiprogramming System. Commun. ACM 1968, 11, 341-346.

(89) Parnas, D. L. On the Criteria to Be Used in Decomposing Systems into Modules. Commun. ACM 1972, 15, 1053-1058.

(90) Frediani, L.; Cammi, R.; Corni, S.; Tomasi, J. A Polarizable Continuum Model for Molecules at Diffuse Interfaces. J. Chem. Phys. 2004, 120, 3893-3907.

(91) Harbrecht, H.; Randrianarivony, M. Wavelet BEM on Molecular Surfaces: Parametrization and Implementation. Computing 2009, 86, 1-22.

(92) Weijo, V.; Randrianarivony, M.; Harbrecht, H.; Frediani, L. Wavelet Formulation of the Polarizable Continuum Model. J. Comput. Chem. 2010, 31, 1469-1477.

(93) Harbrecht, H.; Randrianarivony, M. Wavelet BEM on Molecular Surfaces: Solvent Excluded Surfaces. Computing 2011, 92, 335-364. 
(94) Sumathi, K.; Balasubramanian, K. Electronic States and Potential Energy Surfaces of $\mathrm{H}_{2} \mathrm{Te}, \mathrm{H}_{2} \mathrm{Po}$, and their Positive Ions. J. Chem. Phys. 1990, 92, 6604-6619.

(95) Sadlej, A. J. Medium-Size Polarized Basis Sets for High-Level-Correlated Calculations of Molecular Electric Properties. Theor. Chem. Acc. 1992, 81, 339-354.

(96) Norman, P.; Schimmelpfennig, B.; Ruud, K.; Jensen, H. J. A.; Ågren, H. Relativistic Effects on Linear and Nonlinear Polarizabilities Studied by Effective-Core Potential, Douglas-Kroll, and Dirac-Hartree-Fock Response Theory. J. Chem. Phys. 2002, 116, 6914-6923.

(97) Kellö, V.; Sadlej, A. J. Medium-Size Polarized Basis Sets for High-Level-Correlated Calculations of Molecular Electric Properties. Theor. Chem. Acc. 1992, 83, 351-366.

(98) Alekseyev, A. B.; Liebermann, H.-P.; Wittig, C. On the Ultraviolet Photodissociation of $\mathrm{H}_{2}$ Te. J. Chem. Phys. 2004, 121, 9389-9395.

(99) Vidal, L. N.; Vazquez, P. A. Frequency Dependent Raman Scattering Activities of $\mathrm{BeH}_{2}, \mathrm{MgH}_{2}, \mathrm{CaH}_{2}, \mathrm{SrH}_{2}$, and $\mathrm{H}_{2} \mathrm{O}, \mathrm{H}_{2} \mathrm{~S}, \mathrm{H}_{2} \mathrm{Se}, \mathrm{H}_{2} \mathrm{Te}$, Evaluated by the Ab Initio Relativistic Four Component Method Dirac-Hartree-Fock. Chem. Phys. 2006, 321, 209-214.

(100) Dubillard, S.; Rota, J.-B.; Saue, T.; Fægri, K. Bonding Analysis Using Localized Relativistic Orbitals: Water, the Ultrarelativistic Case and the Heavy Homologues $\mathrm{H}_{2} \mathrm{X}(\mathrm{X}=\mathrm{Te}, \mathrm{Po}$, eka-Po). J. Chem. Phys. 2006, 124, 154307-154321.

(101) Ndoye, C. A. A.; Daniel, C. Electronic Absorption Spectroscopy of $\mathrm{H}_{2} \mathrm{X}$ (X = O, Te, Po): Theoretical Treatment of Spin-Orbit Effects. Chin. J. Chem. Phys. 2009, 22, $171-177$.

(102) Adamo, C.; Barone, V. Toward Reliable Density Functional Methods Without Adjustable Parameters: the PBE0 Model. J. Chem. Phys. 1999, 110, 6158-6170. 
(103) Visscher, L.; Saue, T. Approximate Relativistic Electronic Structure Methods Based on the Quaternion Modified Dirac Equation. J. Chem. Phys. 2000, 113, 3996-4002.

(104) Dunning, T. H., Jr. Gaussian Basis Sets for Use in Correlated Molecular Calculations.I. The Atoms Boron Through Neon and Hydrogen. J. Chem. Phys. 1989, 90, 1007-1023.

(105) Woon, D. E.; Dunning, T. H., Jr. Gaussian Basis Sets for Use in Correlated Molecular Calculations.III. The Atoms Aluminum Through Argon. J. Chem. Phys. 1993, 98, 1358-1371.

(106) Dyall, K. G. Relativistic and Nonrelativistic Finite Nucleus Optimized Double Zeta Basis Sets for the 4p, 5p and 6p Elements. Theor. Chem. Acc. 1998, 99, 366-371.

(107) Dyall, K. G. Relativistic and Nonrelativistic Finite Nucleus Optimized Double Zeta Basis Sets for the 4p, 5p and 6p Elements (Theor Chem Acc (1998) 99:366-371): Addendum. Theor. Chem. Acc. 2002, 108, 365-365.

(108) Dyall, K. G. Relativistic Quadruple-Zeta and Revised Triple-Zeta and Double-Zeta Basis Sets for the 4p, 5p, and 6p Elements. Theor. Chem. Acc. 2006, 115, 441-447.

(109) DALTON, a molecular electronic structure program, Release Dalton2011 (2011), see http://daltonprogram.org/.

(110) Bakken, V.; Helgaker, T. The efficient optimization of molecular geometries using redundant internal coordinates. J. Chem. Phys. 2002, 117, 9160-9174.

(111) Mantina, M.; Chamberlin, A. C.; Valero, R.; Cramer, C. J.; Truhlar, D. G. Consistent van der Waals Radii for the Whole Main Group. J. Phys. Chem. A 2009, 113, 58065812.

(112) Tomasi, J.; Persico, M. Molecular Interactions in Solution: An Overview of Methods Based on Continuous Distributions of the Solvent. Chem. Rev. 1994, 94, 2027-2094. 
(113) Bondi, A. van der Waals Volumes and Radii. J. Phys. Chem. 1964, 68, 441-451.

(114) Jaszuński, M.; Rizzo, A.; Ruud, K. In Handbook of Computational Chemistry; Leszczynski, J., Ed.; Springer Science+Business Media B.V., 2012; pp 361-441.

(115) Onsager, L. Electric Moments of Molecules in Liquids. J. Am. Chem. Soc. 1936, 58, $1486-1493$.

(116) Wheeler, S. E.; Houk, K. N. Through-Space Effects of Substituents Dominate Molecular Electrostatic Potentials of Substituted Arenes. J. Chem. Theory Comput. 2009, 5, 2301-2312.

(117) Raptis, S. G.; Papadopoulos, M. G.; Sadlej, A. J. The Correlation, Relativistic, and Vibrational Contributions to the Dipole Moments, Polarizabilities, and First and Second Hyperpolarizabilities of ZnS, CdS, and HgS. J. Chem. Phys. 1999, 111, 7904-7915.

(118) da Silva, C. O.; Mennucci, B.; Vreven, T. Density functional study of the optical rotation of glucose in aqueous solution. J. Org. Chem. 2004, 69, 8161-4.

(119) Amovilli, C.; Mennucci, B. Self-Consistent-Field Calculation of Pauli Repulsion and Dispersion Contributions to the Solvation Free Energy in the Polarizable Continuum Model. J Phys Chem B 1997, 101, 1051-1057.

(120) Pierotti, R. Scaled Particle Theory of Aqueous and Non-aqueous Solutions. Chem. Rev. 1976, 76, 717-726.

(121) Abbotto, A.; Beverina, L.; Bradamante, S.; Facchetti, A.; Klein, C.; Pagani, G. A.; Redi-Abshiro, M.; Wortmann, R. A distinctive example of the cooperative interplay of structure and environment in tuning of intramolecular charge transfer in second-order nonlinear optical chromophores. Chemistry 2003, 9, 1991-2007.

(122) Mikkelsen, K. V.; Luo, Y.; Agren, H.; Jorgensen, P. Sign change of hyperpolarizabilities of solvated water. J Chem Phys 1995, 102, 9362. 
(123) Mikkelsen, K. V.; Jorgensen, P.; Jensen, H. J. A. A multiconfiguration self-consistent reaction field response method. J Chem Phys 1994, 100, 6597-6607. 


\section{Graphical TOC Entry}

Differential molecular electrostatic potential (MEP) for $\mathrm{H}_{2} \mathrm{Po}$ calculated at the DFT/PBEO level of theory using the Dirac-Coulomb Hamiltonian. The difference is taken between the MEP calculated in water solution, using the PCM, and in vacuo. The differential MEP is in the range $[-0.01,0.01] E_{\mathrm{h}} e^{-1}$ and is color-coded from red to blue. The differential MEP is mapped on 25 different isodensity surfaces, ranging from $[-0.2,0.2] e a_{0}^{-3}$. The isodensity contours are calculated at the Dirac-Coulomb DFT/PBE0 level of theory in vacuo. The contour lines resulting from slicing the plot on the molecular plane are also reported, color-coded according to the differential MEP scale.

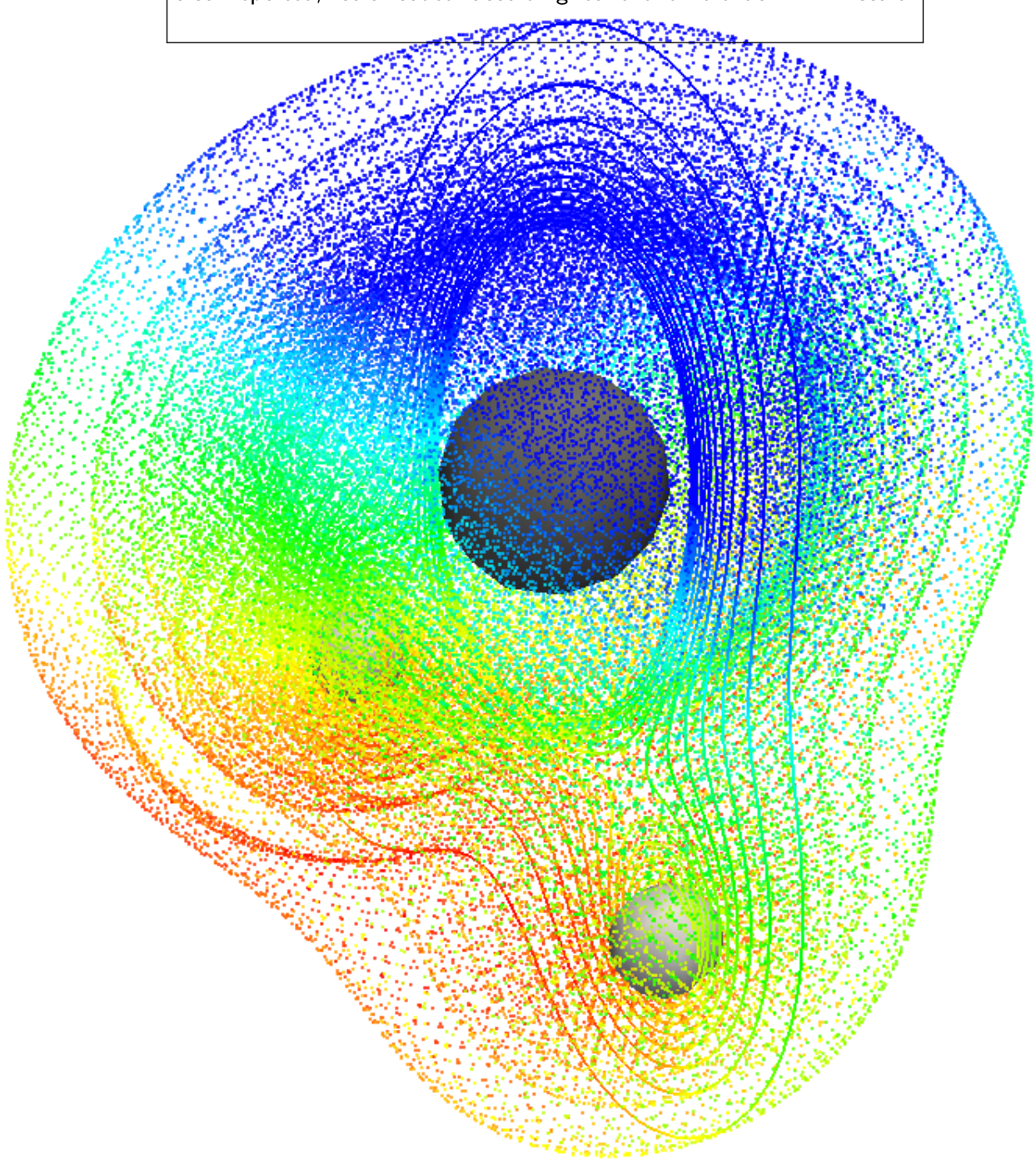

